\title{
Integrative analysis of Iso-Seq and RNA-seq data reveals transcriptome complexity and differentially expressed transcripts in sheep tail fat
}

\author{
Zehu Yuan ${ }^{1}$, Ling Ge ${ }^{2}$, Jingyi Sun ${ }^{3}$, Weibo Zhang ${ }^{2}$, Shanhe Wang $^{2}$, Xiukai Cao ${ }^{1}$, Wei Sun ${ }^{\text {Corresp. } 1,2}$ \\ 1 Joint International Research Laboratory of Agriculture and Agri-Product Safety of Ministry of Education, Yangzhou University, Yangzhou, China \\ 2 College of Animal Science and Technology, Yangzhou University, Yangzhou, China \\ 3 College of Veterinary Medicine, Yangzhou University, Yangzhou, China \\ Corresponding Author: Wei Sun \\ Email address: dkxmsunwei@163.com
}

\section{Background}

Nowadays, both customers and producers prefer thin-tailed fat sheep. To effectively breed for this phenotype, it is important to identify candidate genes and uncover the genetic mechanism related to tail fat deposition in sheep. Accumulating evidence suggesting that post-transcriptional modification events of precursor-messenger RNA (pre-mRNA), including alternative splicing (AS) and alternative polyadenylation (APA), may regulate tail fat deposition in sheep. Differentially expressed transcripts (DETs) analysis is a way to identify candidate genes related to tail fat deposition. However, due to the technological limitation, post-transcriptional modification events in the tail fat of sheep and DETs between thin-tailed and fat-tailed sheep remains unclear.

\section{Methods}

In the present study, we applied pooled PacBio isoform sequencing (Iso-Seq) to generate transcriptomic data of tail fat tissue from six sheep (three thin-tailed sheep and three fat-tailed sheep). By comparing with reference genome, potential gene loci and novel transcripts were identified. Post-transcriptional modification events, including AS and APA, and IncRNA in sheep tail fat were uncovered using pooled IsoSeq data. Combining Iso-Seq data with and six RNA-sequencing (RNA-Seq) data, DETs between thin- and fat-tailed sheep were identified. Protein protein interaction (PPI) network, Gene Ontology (GO) and Kyoto Encyclopedia of Genes and Genomes (KEGG) enrichment analyses were implemented to investigate the potential functions of DETs.

\section{Results}

In the present study, we revealed the transcriptomic complexity of the tail fat of sheep, result in 9001 potential novel gene loci, 17834 AS events, 5791 APA events, and 3764 IncRNAs. Combining Iso-Seq data with RNA-Seq data, we identified hundreds of DETs between thin- and fat-tailed sheep. Among them, 21 differentially expressed IncRNAs, such as ENSOART00020036299, ENSOART00020033641, ENSOART00020024562, ENSOART00020003848 and 9.53.1 may regulate tail fat deposition. Many novel transcripts were identified as DETs, including 15.527.13 (DGAT2), 13.624.23 (ACSS2), 11.689.28 (ACLY), 11.689.18 (ACLY), 11.689.14 (ACLY), 11.660.12 (ACLY), 22.289.6 (SCD), 22.289.3 (SCD) and 22.289.14 $(S C D)$. Most of the identified DETs have been enriched in GO and KEGG pathways related to extracellular matrix (ECM). Our result revealed the transcriptome complexity and identified many candidate 
transcripts in tail fat, which could enhance the understanding of molecular mechanisms behind tail fat deposition. 
2 Integrative analysis of Iso-Seq and RNA-seq data

3 reveals transcriptome complexity and differentially

4 expressed transcripts in sheep tail fat

5

6

7

8 9

Zehu Yuan ${ }^{1}$, Ling $\mathrm{Ge}^{2}$, Jingyi Sun ${ }^{3}$, Weibo Zhang ${ }^{2}$, Shanhe Wang ${ }^{2}$, Xiukai Cao ${ }^{1}$, Wei Sun ${ }^{1,2^{*}}$

${ }^{1}$ Joint International Research Laboratory of Agriculture and Agri-Product Safety of Ministry of Education, Yangzhou University, Yangzhou 225000, China

${ }^{2}$ College of Animal Science and Technology, Yangzhou University, Yangzhou 225000, China

${ }^{3}$ College of Veterinary Medicine, Yangzhou University, Yangzhou 225000, China

\section{Corresponding Author:}

Wei Sun ${ }^{1}$

International cooperation building (7\#), Wenhui Campus, Yangzhou University, Yangzhou 22500, Jiangsu, China

Email address: dkxmsunwei@163.com

\section{Abstract \\ Background}

Nowadays, both customers and producers prefer thin-tailed fat sheep. To effectively breed for this phenotype, it is important to identify candidate genes and uncover the genetic mechanism related to tail fat deposition in sheep. Accumulating evidence suggesting that post-transcriptional modification events of precursor-messenger RNA (pre-mRNA), including alternative splicing (AS) and alternative polyadenylation (APA), may regulate tail fat deposition in sheep. Differentially expressed transcripts (DETs) analysis is a way to identify candidate genes related to tail fat deposition. However, due to the technological limitation, post-transcriptional modification events in the tail fat of sheep and DETs between thin-tailed and fat-tailed sheep remains unclear.

\section{Methods}

In the present study, we applied pooled PacBio isoform sequencing (Iso-Seq) to generate transcriptomic data of tail fat tissue from six sheep (three thin-tailed sheep and three fat-tailed sheep). By comparing with reference genome, potential gene loci and novel transcripts were identified. Post-transcriptional modification events, including AS and APA, and lncRNA in sheep tail fat were uncovered using pooled Iso-Seq data. Combining Iso-Seq data with and six RNAsequencing (RNA-Seq) data, DETs between thin- and fat-tailed sheep were identified. Protein 
40 protein interaction (PPI) network, Gene Ontology (GO) and Kyoto Encyclopedia of Genes and

41 Genomes (KEGG) enrichment analyses were implemented to investigate the potential functions

42 of DETs.

43

44

\section{Results}

In the present study, we revealed the transcriptomic complexity of the tail fat of sheep, result in

46

47

48

49

50

51

52

53

54

55

56

57

58

59

60

61

62

63

64

65

66

67

68

69

70

71

72

73

74

75

76

77

78

79 9001 potential novel gene loci, 17834 AS events, 5791 APA events, and 3764 lncRNAs. Combining Iso-Seq data with RNA-Seq data, we identified hundreds of DETs between thin- and fat-tailed sheep. Among them, 21 differentially expressed lncRNAs, such as ENSOART00020036299, ENSOART00020033641, ENSOART00020024562, ENSOART00020003848 and 9.53.1 may regulate tail fat deposition. Many novel transcripts were identified as DETs, including 15.527 .13 (DGAT2), 13.624.23 (ACSS2), 11.689.28 (ACLY), 11.689.18 (ACLY), 11.689.14 (ACLY), 11.660.12 (ACLY), 22.289.6 (SCD), 22.289.3 (SCD) and 22.289.14 $(S C D)$. Most of the identified DETs have been enriched in GO and KEGG pathways related to extracellular matrix (ECM). Our result revealed the transcriptome complexity and identified many candidate transcripts in tail fat, which could enhance the understanding of molecular mechanisms behind tail fat deposition.

\section{Introduction}

Post-transcriptional modification events of precursor-messenger RNA (pre-mRNA), including alternative splicing (AS) and alternative polyadenylation (APA), have attracted an increasing interest (Baralle \& Giudice 2017; Di Giammartino et al. 2011; Gruber \& Zavolan 2019; Kornblihtt et al. 2013; Wang et al. 2015). In livestock, accumulated evidence suggests that AS (Fang et al. 2020; Leal-Gutierrez et al. 2020; Xiang et al. 2018; Yuan et al. 2021) and APA (Deng et al. 2020; Jin et al. 2021) could contribute to the formation of important economic traits. For example, both AS (Motter et al. 2021) and APA (Nattrass et al. 2014) in calpastatin (CAST) were associated with meat tenderness in beef. RNA-sequencing (RNA-seq) is a way to accurately qualify the AS events, e.g., using the intron excision ratio (Li et al. 2018c). Unfortunately, it is unlikely to obtain the fulllength transcripts and quantify the transcripts by RNA-seq because it is difficult to assemble fulllength transcripts based on short reads and to exactly assign a short read to a certain transcript (Kovaka et al. 2019), which hamper the discovery of post-transcriptional events. The thirdgeneration sequencing technology, such as PacBio isoform sequencing (Iso-Seq), which can directly produce full length transcripts (Rhoads \& Au 2015), providing an opportunity to discover novel genes and novel isoforms (Au et al. 2013; Beiki et al. 2019; Chen et al. 2017; Karlsson \& Linnarsson 2017) and to investigate the transcriptome complexity in mammals (Deng et al. 2020; Li et al. 2018b). In addition, the results from the previous study suggested that integrative analysis of Iso-Seq and RNA-seq data could accurately quantify the abundance of transcripts (Chao et al. 2019; Li et al. 2017). Thus, using Iso-Seq and RNA-seq could accurately identify the posttranscriptional modification events and differential expression isoforms. 
80 Hu sheep, a short fat-tailed sheep breed, is one of the most popular sheep breeds in China because

81 of its high fecundity. In an intensive production system, Hu sheep is usually used as ewe crossing 82 with elite ram breeds, e.g., Dorper, to produce meat. Backcross ((Dorper $\times \mathrm{Hu}) \times \mathrm{Hu}, \mathrm{DHH})$ and 83 grading up (Dorper $\times($ Dorper $\times \mathrm{Hu}), \mathrm{DDH})$ are two common hybridization methods in sheep meat 84 production. The weight of the tail fat of their hybrid offspring is positively related to the 85 composition of the $\mathrm{Hu}$ sheep genome. Nowadays, both customers and producers prefer a small 86 amount of tail fat. To breed for this phenotype, it is important to identify the candidate genes linked 87 with tail fat and understand the underlying mechanisms of fat deposition. 88

89

90

91

92

93

94

95

96

97

98

99

100

101

102

103

104

105

106

107

108

109

110

111

112

113

114

115

116

117

118

Many studies have attempted to identify the candidate gene associated with tail fat and understand the underlying mechanisms of fat deposition (Ahbara et al. 2018; Amane et al. 2020; Baazaoui et al. 2021; Bakhtiarizadeh \& Alamouti 2020; Dong et al. 2020; Han et al. 2021; Li et al. 2018a; Mastrangelo et al. 2019a; Mastrangelo et al. 2019b; Moioli et al. 2015; Moradi et al. 2012; Wei et al. 2015; Xu et al. 2017; Yuan et al. 2017; Zhang et al. 2019; Zhao et al. 2020; Zhi et al. 2018; Zhu et al. 2016). Previous studies suggested that microRNAs (Miao et al. 2015a; Pan et al. 2018), lncRNAs (Bakhtiarizadeh \& Salami 2019; He et al. 2020) and mRNAs (Bakhtiarizadeh et al. 2019; Kang et al. 2017a; Miao et al. 2015b; Wang et al. 2014) may regulate tail fat deposition in sheep. These results contribute to understanding the underlying mechanisms of fat deposition. However, due to the technological limitation, post-transcriptional modification events (e.g., AS and APA) in the tail fat of sheep remains unclear, which might play an important role in tail fat deposition. In the current study, Iso-Seq was used to uncover post-transcriptional modifications in sheep tail fat and combined with RNA-Seq to investigate differential expressed transcripts (DETs) in tail fat between DHH and DDH. Our results could reveal transcriptome complexity in sheep tail fat and characterize DETs between DHH and DDH sheep.

\section{Materials \& Methods}

\section{Animal tissues collection and RNA extraction}

After wearing (2-month old), all animals were fed to 6-month old in house with complete formula granulated feed (DafengGe Feed Technology Co., Ltd.) at Suyang Sheep Industry Co., Ltd (Fengxian, Jiangsu, China). All animals were eating and drinking freely. In total, six unrelated 6month old male sheep ( $\mathrm{DDH}=3, \mathrm{DHH}=3$ ) with similar live weight (Table 1) were selected to slaughter for sampling. Animal experiments were approved by the Experimental Animal Ethical Committee of Yangzhou University (NO.202103294).

After slaughter, tail fat tissues were sampled within 30 minutes. Each fat sample was packed into a $1.5 \mathrm{ml}$ cryotube. All the fat samples were quickly frozen in liquid nitrogen and stored at $-80{ }^{\circ} \mathrm{C}$. Total RNA was extracted from the tail fat using Trizol reagent Kit (TaKaRa, USA). The extraction protocol strictly follows the instructions of Kit. The quality of RNA samples was evaluated by Nanodrop 2000 (Thermo Scientific $^{\mathrm{TM}}$ ) and 2100 Bioanalyzer (Agilent Technologies, Waldbronn, 
119 Germany). After quality control, all the RNA samples with RNA Integrity Number (RIN) greater

120

121

122

123

124

125

126

127

128

129

130

131

132

133

134

135

136

137

138

139

140

141

142

143

144

145

146

147

148

149

150

151

152

153

154

155

156

157 158

than 7.0 and $28 \mathrm{~S} / 18 \mathrm{~S}$ ratio greater than 1.0 were used for sequencing.

\section{Library Construction and Sequencing of RNA-sequencing}

Library construction and sequencing of RNA-seq of six RNA samples were performed by a commercial sequencing service company (Frasergene Technology Co., Ltd, Wuhan, China). Briefly, the MGIEasy RNA Directional Library Prep Kit (MGI) and $1 \mu \mathrm{g}$ of total RNA were used for library construction. Then, the library was sequenced on the MGISEQ-2000 platform. All sequencing data have been deposited in National Center for Biotechnology Information (NCBI) Short Read Archive (SRA) database and can be accessed under the BioProject accession number PRJNA745517.

\section{Iso-Seq Library Construction and Sequencing}

Six RNA samples $(\mathrm{DDH}=3, \mathrm{DHH}=3)$ were pooled in equal quantities for Iso-Seq. Library preparation and Iso-Seq were performed by a commercial sequencing service company (Frasergene Technology Co., Ltd, Wuhan, China). Briefly, the SMARTerTM PCR cDNA synthesis kit (Takara Biotechnology, Dalian, China) was used to reverse-transcribed RNA into cDNA. Then, PCR Amplification was implemented by using 12-14 PCR cycles. PCR products were purified by the AMPure PB magnetic beads (Beckman Coulter, CA, USA). The BluePippinTM Size Selection System (Sage Science, MA, USA) was used for the size selection $(>1 \mathrm{~kb}$ ). Pacific Biosciences DNA Template Prep Kit 2.0 (Pacific Biosciences, CA, USA) was used for SMRTbell library construction. Agilent Bioanalyzer 2100 system (Agilent Technologies, CA, USA) and Qubit fluorometer 2.0 (Life Technologies, CA, USA) were used for quality accession of the library. Then, the library was sequenced on the PacBio sequencing platform with 10h sequencing movies.

\section{Iso-Seq data processing}

The pipeline of bioinformatics analysis was shown in Fig.1. The raw reads of Iso-Seq were preprocessed using SMRT Link v8.0 (https://www.pacb.com/wp-content/uploads/SMRT-LinkRelease-Notes-v8.0.pdf). The adapter of polymerase reads was remove to get Subreads. Circular Consensus sequencing (CCS) reads were obtained from the Subreads using the following parameters: minimum subread length $=50$, maximum subread length $=15000$, minimum number of passes $=3$, and minimum predicted accuracy $=0.99$. Then, CCS were classified into full length reads by lima software. Full-length non-chimeric reads (FLNCs) were full-length CCS reads with 5' and 3' cDNA primers and polyA. The LoRDEC v0.9 software (Salmela \& Rivals 2014) was used for FLNC correction using the high-quality short reads with the default parameters. The quality of RNA-seq data was examined using SOAPnuke v2.1.0 (Chen et al. 2018). FLNCs were aligned to the sheep reference genome (http://ftp.ensembl.org/pub/release102/fasta/ovis_aries_rambouillet/dna/) using GMAP (Wu \& Watanabe 2005).

PeerJ reviewing PDF | (2021:07:63246:1:1:NEW 9 Sep 2021) 
159 Novel genes and isoforms identification

160 Based on the results from alignments, FLNCs sharing the same splicing event were merged into 161 one isoform. The isoforms with $5^{\prime}$ terminal region degraded were excluded for further analysis. 162 Isoforms meet one of the following conditions were kept: 1) isoform was supported by at least two 163 FLNC; 2) isoform was supported by one FLNC whose Percentage Identity (PID) was greater than

164

165

166

167

168

169

170

171

172

173

174

175

176

177

178

179

180

181

182

183

184

185

186

187

188

189

190

191

192

193

194

195

196

197

198

199

$99 \%$; 3) all splicing sites in an isoform were fully supported by short reads; 4) all splicing junctions in an isoform were annotated by the reference genome. Isoforms overlapped over $20 \%$ of their length on the same strand were regarded as transcribing from the same gene locus (Wang et al. 2021). A gene locus was defined as a novel gene if it overlaps less than $20 \%$ of length with known genes. A novel isoform was defined as an isoform with a final splice site of $3^{\prime}$ ends changed or a new intron (exon) emerged.

\section{Functional annotation of isoforms}

To better understand the potential function of isoforms, they were aligned to Gene Ontology (GO) (Ashburner et al. 2000), Swiss-Prot (Gasteiger et al. 2001), NCBI non-redundant proteins (NR) and Cluster of Orthologous Groups of proteins (COG/KOG) (Tatusov et al. 2003) database using Diamond (Buchfink et al. 2015). Isoforms were also aligned to KEGG (https://www.genome.jp/kegg/) database using KOBAS (Xie et al. 2011).

\section{IncRNA prediction}

Novel isoforms with lengths greater than $200 \mathrm{nt}$ were blast against the NR, KOG/KO and SwissProt to remove transcripts with coding potential. A transcript with above annotated information was removed for further lncRNA prediction. Multiple software, including CPC 2.0 beta (default parameter) (Kang et al. 2017b), CNCI v2 (-m ve) (Sun et al. 2013), PLEK v1.2 (-minlength 200) (Li et al. 2014) and CPAT v1.2.4 (default parameter) (Wang et al. 2013), were used to predict lncRNAs from novel isoforms.

\section{APA and AS analysis}

The APA sites for each gene loci were detected using the TAPIS pipeline (Abdel-Ghany et al. 2016). AStalavista v3.2 software was used to detect AS events with default parameters (Foissac \& Sammeth 2007).

\section{DETs detection}

Reference genome annotation file and PacBio novel isoforms were merged to make a novel annotation file. The clean reads of each RNA-Seq library were aligned to the novel annotation file using the Bowtie2 software (-q --sensitive --dpad 0 --gbar 99999999 --mp 1,1 --np 1 --score-min L,0,-0.1 -I 1 -X 1000 --no-mixed --no-discordant -p 6) (Langmead \& Salzberg 2012). The RSEM software was used to quantify transcripts (Li \& Dewey 2011). DESeq2 R package (Love et al. 2014) was used to identify DETs between thin and fat-tailed sheep samples. Transcripts with the false discovery rate (FDR) smaller than 0.05 and fold change greater than 2 were defined as significant DETs. The correlation between differentially expressed lncRNAs and DETs which

Peer] reviewing PDF | (2021:07:63246:1:1:NEW 9 Sep 2021) 
200

201

202

203

204

205

206

207

208

209

210

211

212

213

214

215

216

217

218

219

220

221

222

223

224

225

226

227

228

229

230

231

232

233

234

235

236

237

238

239

were transcribed from tail fat related candidate genes was implemented using the ggcor R package (Houyun Huang et al. 2020).

\section{Functional annotation of DETs}

To extract the potential function of DETs, DETs were blast to GO database using Diamond (Buchfink et al. 2015) and KEGG database using KOBAS (Xie et al. 2011). FDR $<0.05$ was regarded as the significant threshold. The GO enrichment results were visualized by the GOplot 1.0.2 R package (Walter et al. 2015). To explore the interaction between DETs and transcripts which were transcribed from the candidate genes linked with tail fat deposition, protein-protein interaction (PPI) networking analysis was implemented using the STRING database (Szklarczyk et al. 2017). The result of PPI was visualized by the Cytoscape v3.8.3 software (Shannon et al. 2003).

\section{Results}

\section{PacBio Iso-Seq and bioinformatics analysis}

In total, 487,822 polymerase reads were generated by Iso-Seq (Table 2). After quality control, $30,305,694$ filtered subreads with a mean length of 1,474 bp and 360,901 CCS reads with an average depth of 71 passes were produced (Table 2). Finally, 271,718 FLNCs with an average length of 1,754 bp were used for further analysis (Table 2).

\section{Gene loci and isoforms detection}

All FLNCs were corrected for sequencing errors and alignment position. According to the alignment position of each FLNC, gene loci and isoforms were identified. In total, 20,041 gene loci (11,040 known gene loci and 9,001 potential novel gene loci) were identified by Iso-Seq (Fig.2A). A total of 7,187 (35.86\%) detected genes generate two or more isoforms (Fig.2B). Interestingly, the LIPE (lipase E, hormone-sensitive type) transcribed the greatest number of transcripts (113 novel transcripts and 3 known transcripts). In sheep tail fat, the majority of novel isoforms are transcribed from annotated genes (Fig. 2C). All novel gene loci and novel isoforms were added to the reference annotation file (Generic Feature Format, GFF, Table S1).

To elucidate the potential function of novel isoforms transcribed from novel genes, isoforms were annotated to public databases, including Gene Ontology (GO) (Ashburner et al. 2000), Swiss-Prot (Gasteiger et al. 2001), NCBI non-redundant proteins (NR), Cluster of Orthologous Groups of proteins (COG/KOG) (Tatusov et al. 2003) and Kyoto Encyclopedia of Genes and Genomes (KEGG, https://www.genome.jp/kegg/) database (Table S2). In total, above half novel transcripts $(5762,58.87 \%)$ were annotated at least one public database, including 5,697 (58.21\%) in NR, 2,390 (24.42\%) in GO, 3,944 (40.30\%) in KEGG, 351 (3.59\%) in KOG and 1,955 (19.98\%) in Swiss-Prot (Fig. 3).

\section{IncRNA prediction}


240 In the current study, four software were used to predict lncRNAs. Finally, 3764 lncRNAs were 241 predicted by all software (Fig. 4A). The sequence of predicted lncRNAs were documented in Table 242 S3. The length of predicted lncRNAs ranged from 205 to 5698 bp (Fig.4B). The mean length of 243 lncRNAs was 1198 bp.

244

245

246

247

248

249

250

251

252

253

254

255

256

257

258

259

260

261

262

263

264

265

266

267

268

269

270

271

272

273

274

275

276

277

278

279

\section{Alternative polyadenylation events detection}

TAPIS pipeline was used to detect APA events in tail fat. In total, 5791 detected genes had at least one poly(A) site (Fig.5A, Table S4). Of them, 4129 genes contained a single poly(A) site (Fig.5A). The remaining $1662(28.70 \%)$ genes contained two or more poly(A) sites (Fig.5A). The ENSOARG00020025751 (ASPH) and ENSOARG00020017413 (unannotated) contained the greatest number of distinct poly(A) sites, which were illustrated in Fig.5B and Fig.5C.

\section{Alternative splicing events detection}

In total, 17834 AS events were detected by Iso-Seq data. AS events were classified into four basic types and others (Fig.6). Among four basic types, exon skipping (4679, 26.24\%) is the most prevalent AS event, followed by alternate acceptor site $(2728,15.30 \%)$, intron retaining (2624, $14.71 \%)$ and alternate donor site $(1409,7.90 \%)$.

\section{Differentially expressed transcripts}

In our study, a total of 464 DETs between thin and fat-tailed samples were identified (Fig.7A, Table S5). The most significant differential transcript was ENSOART00020021670 (elongation factor 1-beta, FDR= 5.79e-19). Among these DETs, 21 DETs are differentially expressed lncRNAs, including 14 known lncRNAs and seven novel lncRNAs. The correlation between differentially expressed lncRNAs and DETs which transcribed from tail fat related candidate genes, including diacylglycerol O-acyltransferase 2 (DGAT2) (Bakhtiarizadeh \& Alamouti 2020), acetyl-CoA carboxylase alpha $(A C A C A)$ (Bakhtiarizadeh \& Alamouti 2020; Bakhtiarizadeh \& Salami 2019), ATP citrate lyase (ACLY) (Bakhtiarizadeh \& Alamouti 2020), fatty acid synthase $(F A S N)$ (Bakhtiarizadeh \& Alamouti 2020), stearoyl-CoA desaturase (SCD) (Kang et al. 2017a), and acyl-CoA synthetase short chain family member 2 (ACSS2) (Guangli et al. 2020), was investigated. The result suggests that 20 out of 21 lncRNAs (except lncRNA ENSOART00020017088) were significantly correlated with at least one transcripts (Fig.7B).

\section{Functional annotation of DETs}

PPI analysis was implemented to identify functional similarity between the DETs. All of the networks were documented in Table S6. Here, we highlight an example. ACACA is a candidate gene related to tail fat deposition (Bakhtiarizadeh \& Alamouti 2020; Bakhtiarizadeh \& Salami 2019). In the current study, we found ten DETs $(11.99 .3,11.689 .28,1.689 .18,11.265 .3$, ENSOART00020040904, 11.99.15, 11.689.14, 13.624.23, ENSOART00020006006, and 15.327.19) directly interactive with ENSOART00020037575 which was transcribed from $A C A C A$ (Fig.8A). To further understand the function of DETs, GO and KEGG enrichment analyses were 
280 implemented. FDR $<0.05$ was regarded as the significant threshold. All DETs were significantly 281 enriched in $38 \mathrm{GO}$ terms (Fig.8B, Table S7). The most significant enriched GO term is 282 extracellular matrix structural constituent (GO:0005201, FDR=1.87e-4, Fig.8B), including three 283 annotated transcripts (ENSOART00020027615, ENSOART00020019556 and 284 ENSOART00020033210) and nine novel transcripts $(11.236 .9,10.185 .4,4.380 .12,4.380 .22$, 285 10.185.6, 24.240.21, 3.1134.2, 2.407.17, and 10.185.7). Detected DETs were significantly 286 enriched in six KEGG pathways (Fig.8C, Table S8). The most significant enriched pathway is 287 Extracellular Matrix-receptor (ko04512), including three known transcripts 288 (ENSOART00020039107, ENSOART00020027615, and ENSOART00020006511) and 11 novel 289 transcripts $(4.380 .22,11.236 .16,3.143 .5,10.185 .4,10.185 .6,10.185 .7,4.380 .12,12.528 .5$, 290 1.2008.6, 1.2007.14, and 11.236.9). The most significant GO term and KEGG pathway are 291 extracellular matrix related indicating extracellular matrix play an important role in tail fat 292 deposition.

293

294

295

296

297

298

299

300

301

302

303

304

305

306

307

308

309

310

311

312

313

314

315

316

317

318

319

\section{Discussion}

In the current study, PacBio Iso-Seq was applied to uncovering the complexity of the transcriptome profile of the tail fat. Using Iso-Seq, 9001 potential novel genes and 36777 novel transcripts were detected (Fig.2). The most notable gene was LIPE (lipase E, hormone-sensitive type) with 116 transcripts (113 novel transcripts and 3 known transcripts). The main function of hormonesensitive lipase in adipose is to break triacylglycerol into fatty acids (Recazens et al. 2020). In humans, four exons (called exon B, A, T2 and T1) are alternatively used to produce different LIPE transcripts (Recazens et al. 2020). Similar AS patterns have also been found in rats, ewe, chickens, and fish (grass carp) (Bonnet et al. 1998; Cecilia et al. 1988; Sun et al. 2017). This suggests that Iso-Seq is promising in identifying novel gene loci and novel isoforms.

LncRNAs were predicted using Iso-Seq data to understand the transcriptome complexity of sheep tail fat. There are previous reports regarding lncRNA regulating tail fat deposition (Bakhtiarizadeh \& Salami 2019; Ma et al. 2018). These previous studies suggest that only a small portion of novel lncRNA identified in sheep tail fat were conserved (Bakhtiarizadeh \& Salami 2019). In the current study, we identified 3764 lncRNA (Fig.4, Table S3) and they could be useful for further investigating how lncRNA regulates tail fat deposition. Among them, 21 differentially lncRNAs were identified (Fig.7B). It has been reported that lincRNA.3473 interactive with $A C A C A$ may regulate fat deposition (Bakhtiarizadeh \& Salami 2019). In the current study, the positive significant correlation between several lncRNAs (ENSOART00020036299, ENSOART00020033641, ENSOART00020024562, ENSOART00020003848 and 9.53.1) and $A C A C A$ indicates that these lncRNAs may play a key role in tail fat deposition. The result that the majority of differential expressed lncRNAs (except lncRNA ENSOART00020017088) were significantly correlated with one or more transcripts transcribed from tail fat linked candidate genes could further validate their potential function in tail fat deposition. However, the genetic mechanism underlying lncRNAs regulate tail fat deposition still needs to be further investigated. 
320 In addition, APA was also investigated. In total, 1662 genes contained at least two poly(A) sites

321

322

323

324

325

326

327

328

329

330

331

332

333

334

335

336

337

338

339

340

341

342

343

344

345

346

347

348

349

350

351

352

353

354

355

356

357

358

359

were detected in sheep tail fat (Fig. 5). APA-mediated gene regulatory functions are tissue-specific (Di Giammartino et al. 2011; Lianoglou et al. 2013; MacDonald 2019; Tian \& Manley 2017). Generally, APA could affect mRNA stability, splicing, localization and translation by alter the sequence of regulatory elements, e.g., miRNA binding sites (Elkon et al. 2013; Tian \& Manley 2017). Although how the APA regulates tail fat deposition still needs further study, our results would be a useful resource for further analysis the function of APA in sheep tail fat.

The main function of AS is to increase the diversity of transcripts (Keren et al. 2010). As a result, transcripts expression abundance may change with different phenotypes. Using integrative analysis of Iso-Seq and RNA-seq data, hundreds of DETs were identified between thin- and fattailed sheep (Fig.7A). One of DETs 15.527.13 was transcribed from DGAT2. DGAT2 has been reported to affect tail fat deposition in sheep (Bakhtiarizadeh \& Alamouti 2020) and back fat deposition in pig (Yin et al. 2012). A novel transcript 13.624.23, also a DET, was transcribed from ACSS2. Four DETs, 11.689.28, 11.689.18, 11.689.14 and 11.660.12, were transcribed from ACLY. $A C S S 2$ and $A C L Y$ have been identified as candidate genes related to tail fat deposition in sheep (Bakhtiarizadeh \& Alamouti 2020; Guangli et al. 2020). Cytosolic acetyl CoA (Ac-CoA), the central precursor for lipid biosynthesis in mammals, is produced by $A C L Y$ from mitochondriaderived citrate or by ACSS2 from acetate (Vysochan et al. 2017). ENSOART00020040904 was transcribed from FASN. Three DETs $(22.289 .6,22.289 .3$ and 22.289.14) were transcribed from $S C D$. ENSOART00020037575 was transcribed from ACACA. FASN, ACACA (Bakhtiarizadeh \& Alamouti 2020) and $S C D$ (Kang et al. 2017a) are candidate genes related to tail fat deposition. These three gene, FASN (Leonard et al. 2004) , SCD (Garcia-Fernandez et al. 2010; Kang et al. 2017a) and $A C A C A$ affect fatty acid composition (Cronan \& Waldrop 2002).These suggests that many novel transcripts were transcribed from well-studied candidate genes linked to lipid synthesis may regulate tail fat deposition, highlighting alternative splicing is a complicated process in regulating tailed fat deposition.

PPI analysis is an effective way to search functional similarity between the DETs. For example, ENSOART00020037575 (transcribed from ACACA) directly interactive with 11.265.3 (transcribed from sterol regulatory element binding transcription factor 1, Srebpl) implying that Srebpl may be linked with tailed fat deposition (Fig.8A). Srebpl has been reported to affect tail traits and lipid metabolism in sheep (Liang et al. 2020), which could validate our predicted result. In addition to PPI analysis, the functionality of DETs were also been investigated by GO and KEGG enrichment analyses. Interestingly, the most significant GO term (GO:0005201, extracellular matrix structural constituent, Fig.8B, Table S7) and KEGG pathway (ko04512, ECMreceptor interaction, Fig.8C, Table S8) is extracellular matrix related. Previous studies suggest that differentially expressed genes (DEGs) between fat-tail tissue of fat-tailed sheep and thin-tailed sheep were significantly enriched in the ECM-receptor interaction pathway (Bakhtiarizadeh et al. 2019; Li et al. 2018a). The studies in cattle and human adipose tissue also suggest that ECM plays

Peerj reviewing PDF | (2021:07:63246:1:1:NEW 9 Sep 2021) 
360

361

362

363

364

365

366

367

368

369

370

371

372

373

374

375

376

377

378

379

380

381

382

383

384

385

386

387

388

389

390

391

392

393

394

395

396

397

398

399

400

401

402

403

an important role in adipogenesis (Casado-Diaz et al. 2017; Lee et al. 2013). Consistent with these results, in the present study, many novel DETs were significantly enriched in ECM-related GO terms and pathways, which suggests that Iso-Seq combined with the RNA-seq is a useful way to identification DETs.

\section{Conclusions}

In the present study, PacBio Iso-Seq was used for producing comprehensive transcriptomic data of tail fat of sheep, result in 9001 potential novel gene loci, 17834 AS events, 5791 APA events and 3764 lncRNAs. Combined with Iso-Seq and RNA-Seq data, hundreds of DETs between thin and fat-tailed sheep were identified. Among them, 21 differentially expressed lncRNAs, such as ENSOART00020036299, ENSOART00020033641, ENSOART00020024562, ENSOART00020003848 and 9.53.1 may regulate tail fat deposition. Many novel transcripts were identified as DETs, including 15.527.13 (DGAT2), 13.624.23 (ACSS2), 11.689.28 (ACLY), 11.689.18 (ACLY), 11.689.14 (ACLY), 11.660.12 (ACLY), 22.289.6 (SCD), 22.289.3(SCD) and 22.289.14 $(S C D)$, highlighting AS is a complicated process in regulating tailed fat deposition. Identified DETs were most significantly enriched in ECM-related GO terms and KEGG pathways suggesting their import roles in lipid metabolism. Our result revealed the transcriptome complexity and identified many candidate transcripts in tail fat, which could enhance the understanding of the mechanisms of tail fat deposition.

\section{Acknowledgments}

None.

\section{References}

Abdel-Ghany SE, Hamilton M, Jacobi JL, Ngam P, Devitt N, Schilkey F, Ben-Hur A, and Reddy AS. 2016. A survey of the sorghum transcriptome using single-molecule long reads. Nat Commun 7:11706 DOI:10.1038/ncomms11706

Ahbara A, Bahbahani H, Almathen F, Al Abri M, Agoub MO, Abeba A, Kebede A, Musa HH, Mastrangelo S, Pilla F, Ciani E, Hanotte O, and Mwacharo JM. 2018. Genome-Wide Variation, Candidate Regions and Genes Associated With Fat Deposition and Tail Morphology in Ethiopian Indigenous Sheep. Front Genet 9:699 DOI:10.3389/fgene.2018.00699

Amane A, Belay G, Nasser Y, Kyalo M, Dessie T, Kebede A, Getachew T, Entfellner JD, Edea Z, Hanotte O, and Tarekegn GM. 2020. Genome-wide insights of Ethiopian indigenous sheep populations reveal the population structure related to tail morphology and phylogeography. Genes Genomics 42:1169-1178 DOI:10.1007/s13258-020-00984-y

Ashburner M, Ball CA, Blake JA, Botstein D, Butler H, Cherry JM, Davis AP, Dolinski K, Dwight SS, Eppig JT, Harris MA, Hill DP, Issel-Tarver L, Kasarskis A, Lewis S, Matese JC, Richardson JE, Ringwald M, Rubin GM, and Sherlock G. 2000. Gene ontology: tool for the unification of biology. The Gene Ontology Consortium. Nat Genet 25:25-29 DOI:10.1038/75556

Au KF, Sebastiano V, Afshar PT, Durruthy JD, Lee L, Williams BA, van Bakel H, Schadt EE, Reijo-Pera RA, Underwood JG, and Wong WH. 2013. Characterization of the human ESC transcriptome by hybrid sequencing. Proc Natl Acad Sci U S A 110:E4821-4830 DOI:10.1073/pnas.1320101110

Peer) reviewing PDF | (2021:07:63246:1:1:NEW 9 Sep 2021) 
404

405

406

407

408

409

410

411

412

413

414

415

416

417

418

419

420

421

422

423

424

425

426

427

428

429

430

431

432

433

434

435

436

437

438

439

440

441

442

443

444

445

446

447

448

449

450

451

452

453

454

Baazaoui I, Bedhiaf-Romdhani S, Mastrangelo S, and Ciani E. 2021. Genome-wide analyses reveal population structure and identify candidate genes associated with tail fatness in local sheep from a semi-aridarea. Animal:100193 DOI:10.1016/j.animal.2021.100193

Bakhtiarizadeh MR, and Alamouti AA. 2020. RNA-Seq based genetic variant discovery provides new insights into controlling fat deposition in the tail of sheep. Sci Rep 10:13525 DOI:10.1038/s41598-020-70527-8

Bakhtiarizadeh MR, and Salami SA. 2019. Identification and Expression Analysis of Long Noncoding RNAs in Fat-Tail of Sheep Breeds. G3 (Bethesda) 9:1263-1276 DOI:10.1534/g3.118.201014

Bakhtiarizadeh MR, Salehi A, Alamouti AA, Abdollahi-Arpanahi R, and Salami SA. 2019. Deep transcriptome analysis using RNA-Seq suggests novel insights into molecular aspects of fat-tail metabolism in sheep. Sci Rep 9:9203 DOI:10.1038/s41598-019-45665-3

Baralle FE, and Giudice J. 2017. Alternative splicing as a regulator of development and tissue identity. Nat Rev Mol Cell Biol 18:437-451 DOI:10.1038/nrm.2017.27

Beiki H, Liu H, Huang J, Manchanda N, Nonneman D, Smith TPL, Reecy JM, and Tuggle CK. 2019. Improved annotation of the domestic pig genome through integration of Iso-Seq and RNA-seq data. BMC Genomics 20:344 DOI:10.1186/s12864-019-5709-y

Bonnet M, Faulconnier Y, Flechet J, Hocquette JF, Leroux C, Langin D, Martin P, and Chilliard Y. 1998. Messenger RNAs encoding lipoprotein lipase, fatty acid synthase and hormone-sensitive lipase in the adipose tissue of underfed-refed ewes and cows. Reprod Nutr Dev 38:297-307 DOI:10.1051/rnd:19980310

Buchfink B, Xie C, and Huson DH. 2015. Fast and sensitive protein alignment using DIAMOND. Nat Methods 12:59-60 DOI:10.1038/nmeth.3176

Casado-Diaz A, Anter J, Muller S, Winter P, Quesada-Gomez JM, and Dorado G. 2017. Transcriptomic Analyses of Adipocyte Differentiation From Human Mesenchymal Stromal-Cells (MSC). J Cell Physiol 232:771-784 DOI:10.1002/jcp.25472

Cecilia H, Todd GK, Karen LS, Gudrun F, Staffan N, Chad GM, John ES, Camilla H, Robert SS, Thuluvancheri M, Aldons JL, Per B, and Michael CS. 1988. Hormone-sensitive lipase: sequence, expression, and chromosomal localization to 19 cent-q13.3. Science 241:1503-1506 DOI:10.1126/science.3420405

Chao Q, Gao ZF, Zhang D, Zhao BG, Dong FQ, Fu CX, Liu LJ, and Wang BC. 2019. The developmental dynamics of the Populus stem transcriptome. Plant Biotechnol J 17:206219 DOI:10.1111/pbi.12958

Chen SY, Deng F, Jia X, Li C, and Lai SJ. 2017. A transcriptome atlas of rabbit revealed by PacBio single-molecule long-read sequencing. Sci Rep 7:7648 DOI:10.1038/s41598017-08138-Z

Chen Y, Chen Y, Shi C, Huang Z, Zhang Y, Li S, Li Y, Ye J, Yu C, Li Z, Zhang X, Wang J, Yang $H$, Fang L, and Chen Q. 2018. SOAPnuke: a MapReduce acceleration-supported software for integrated quality control and preprocessing of high-throughput sequencing data. Gigascience 7:1-6 DOI:10.1093/gigascience/gix120

Cronan JE, and Waldrop GL. 2002. Multi-subunit acetyl-CoA carboxylases. Progress in Lipid Research 41:407-435 DOI:10.1016/s0163-7827(02)00007-3

Deng L, Li L, Zou C, Fang C, and Li C. 2020. Characterization and Functional Analysis of Polyadenylation Sites in Fast and Slow Muscles. Biomed Res Int 2020:2626584 DOI:10.1155/2020/2626584

Di Giammartino DC, Nishida K, and Manley JL. 2011. Mechanisms and consequences of alternative polyadenylation. Mol Cell 43:853-866 DOI:10.1016/j.molcel.2011.08.017

Dong K, Yang M, Han J, Ma Q, Han J, Song Z, Luosang C, Gorkhali NA, Yang B, He X, Ma Y, and Jiang L. 2020. Genomic analysis of worldwide sheep breeds reveals PDGFD as a major target of fat-tail selection in sheep. BMC Genomics 21:800 DOI:10.1186/s12864020-07210-9

Peer] reviewing PDF | (2021:07:63246:1:1:NEW 9 Sep 2021) 
455

456

457

458

459

460

461

462

463

464

465

466

467

468

469

470

471

472

473

474

475

476

477

478

479

480

481

482

483

484

485

486

487

488

489

490

491

492

493

494

495

496

497

498

499

500

501

502

503

504

505

Elkon R, Ugalde AP, and Agami R. 2013. Alternative cleavage and polyadenylation: extent, regulation and function. Nat Rev Genet 14:496-506 DOI:10.1038/nrg3482

Fang L, Cai W, Liu S, Canela-Xandri O, Gao Y, Jiang J, Rawlik K, Li B, Schroeder SG, Rosen BD, Li CJ, Sonstegard TS, Alexander LJ, Van Tassell CP, VanRaden PM, Cole JB, Yu Y, Zhang S, Tenesa A, Ma L, and Liu GE. 2020. Comprehensive analyses of 723 transcriptomes enhance genetic and biological interpretations for complex traits in cattle. Genome Res 30:790-801 DOI:10.1101/gr.250704.119

Foissac S, and Sammeth M. 2007. ASTALAVISTA: dynamic and flexible analysis of alternative splicing events in custom gene datasets. Nucleic Acids Res 35:W297-299 DOI:10.1093/nar/gkm311

Garcia-Fernandez M, Gutierrez-Gil B, Garcia-Gamez E, Sanchez JP, and Arranz JJ. 2010. Detection of quantitative trait loci affecting the milk fatty acid profile on sheep chromosome 22: role of the stearoyl-CoA desaturase gene in Spanish Churra sheep. $J$ Dairy Sci 93:348-357 DOI:10.3168/jds.2009-2490

Gasteiger E, Jung E, and Bairoch A. 2001. SWISS-PROT: connecting biomolecular knowledge via a protein database. Curr Issues Mol Biol 3:47-55 DOI:10.21775/cimb.003.047

Gruber AJ, and Zavolan M. 2019. Alternative cleavage and polyadenylation in health and disease. Nat Rev Genet 20:599-614 DOI:10.1038/s41576-019-0145-z

Guangli Y, Huan Z, Shuhong Z, Zhiqiang L, Fengyi G, Guan W, Qiankun W, Xiangyu L, Yongfeng $Y$, and Ming L. 2020. Identification of the Genetic Basis for the Large-tailed Phenotypic Trait in Han Sheep Through Integrated mRNA and miRNA Analysis of Tail Fat Tissue Samples. Research Square DOI:10.21203/rs.3.rs-107294/v1

Han J, Guo T, Yue Y, Lu Z, Liu J, Yuan C, Niu C, Yang M, and Yang B. 2021. Quantitative proteomic analysis identified differentially expressed proteins with tail/rump fat deposition in Chinese thin- and fat-tailed lambs. PLoS One 16:e0246279 DOI:10.1371/journal.pone.0246279

He X, Wu R, Yun Y, Qin X, Chen L, Han Y, Wu J, Sha L, and Borjigin G. 2020. DOI:10.21203/rs.3.rs-131480/v1

Houyun Huang, Lei Zhou, Jian Chen, and Wei T. 2020. ggcor: Extended tools for correlation analysis and visualization. $R$ package version 0.9.8.

Jin W, Zhu Q, Yang Y, Yang W, Wang D, Yang J, Niu X, Yu D, and Gong J. 2021. AnimalAPAdb: a comprehensive animal alternative polyadenylation database. Nucleic Acids Res 49:D47-D54 DOI:10.1093/nar/gkaa778

Kang D, Zhou G, Zhou S, Zeng J, Wang X, Jiang Y, Yang Y, and Chen Y. 2017a. Comparative transcriptome analysis reveals potentially novel roles of Homeobox genes in adipose deposition in fat-tailed sheep. Sci Rep 7:14491 DOI:10.1038/s41598-017-14967-9

Kang YJ, Yang DC, Kong L, Hou M, Meng YQ, Wei L, and Gao G. 2017b. CPC2: a fast and accurate coding potential calculator based on sequence intrinsic features. Nucleic Acids Res 45:W12-W16 DOI:10.1093/nar/gkx428

Karlsson K, and Linnarsson S. 2017. Single-cell mRNA isoform diversity in the mouse brain. BMC Genomics 18:126 DOI:10.1186/s12864-017-3528-6

Keren H, Lev-Maor G, and Ast G. 2010. Alternative splicing and evolution: diversification, exon definition and function. Nat Rev Genet 11:345-355 DOI:10.1038/nrg2776

Kornblihtt AR, Schor IE, Allo M, Dujardin G, Petrillo E, and Munoz MJ. 2013. Alternative splicing: a pivotal step between eukaryotic transcription and translation. Nat Rev Mol Cell Biol 14:153-165 DOI:10.1038/nrm3525

Kovaka S, Zimin AV, Pertea GM, Razaghi R, Salzberg SL, and Pertea M. 2019. Transcriptome assembly from long-read RNA-seq alignments with StringTie2. Genome Biol 20:278 DOI:10.1186/s13059-019-1910-1

Langmead B, and Salzberg SL. 2012. Fast gapped-read alignment with Bowtie 2. Nat Methods 9:357-359 DOI:10.1038/nmeth.1923

Peer) reviewing PDF | (2021:07:63246:1:1:NEW 9 Sep 2021) 
506

507

508

509

510

511

512

513

514

515

516

517

518

519

520

521

522

523

524

525

526

527

528

529

530

531

532

533

534

535

536

537

538

539

540

541

542

543

544

545

546

547

548

549

550

551

552

553

554

Leal-Gutierrez JD, Elzo MA, and Mateescu RG. 2020. Identification of eQTLs and sQTLs associated with meat quality in beef. BMC Genomics 21:104 DOI:10.1186/s12864-0206520-5

Lee HJ, Jang M, Kim H, Kwak W, Park W, Hwang JY, Lee CK, Jang GW, Park MN, Kim HC, Jeong JY, Seo KS, Kim H, Cho S, and Lee BY. 2013. Comparative Transcriptome Analysis of Adipose Tissues Reveals that ECM-Receptor Interaction Is Involved in the Depot-Specific Adipogenesis in Cattle. PLoS One 8:e66267 DOI:10.1371/journal.pone.0066267

Leonard AE, Pereira SL, Sprecher H, and Huang Y-S. 2004. Elongation of long-chain fatty acids. Progress in Lipid Research 43:36-54 DOI:10.1016/s0163-7827(03)00040-7

Li A, Zhang J, and Zhou Z. 2014. PLEK: a tool for predicting long non-coding RNAs and messenger RNAs based on an improved k-mer scheme. BMC Bioinformatics 15:311 DOI:10.1186/1471-2105-15-311

Li B, and Dewey CN. 2011. RSEM: accurate transcript quantification from RNA-Seq data with or without a reference genome. BMC Bioinformatics 12:323 DOI:10.1186/1471-2105-12323

Li B, Qiao L, An L, Wang W, Liu J, Ren Y, Pan Y, Jing J, and Liu W. 2018a. Transcriptome analysis of adipose tissues from two fat-tailed sheep breeds reveals key genes involved in fat deposition. BMC Genomics 19:338 DOI:10.1186/s12864-018-4747-1

Li Y, Dai C, Hu C, Liu Z, and Kang C. 2017. Global identification of alternative splicing via comparative analysis of SMRT- and Illumina-based RNA-seq in strawberry. Plant J 90:164-176 DOI:10.1111/tpj.13462

Li Y, Fang C, Fu Y, Hu A, Li C, Zou C, Li X, Zhao S, Zhang C, and Li C. 2018b. A survey of transcriptome complexity in Sus scrofa using single-molecule long-read sequencing. DNA Res 25:421-437 DOI:10.1093/dnares/dsy014

Li YI, Knowles DA, Humphrey J, Barbeira AN, Dickinson SP, Im HK, and Pritchard JK. 2018c. Annotation-free quantification of RNA splicing using LeafCutter. Nat Genet 50:151-158 DOI:10.1038/s41588-017-0004-9

Liang C, Qiao L, Han Y, Liu J, Zhang J, and Liu W. 2020. Regulatory Roles of SREBF1 and SREBF2 in Lipid Metabolism and Deposition in Two Chinese Representative Fat-Tailed Sheep Breeds. Animals (Basel) 10 DOI:10.3390/ani10081317

Lianoglou S, Garg V, Yang JL, Leslie CS, and Mayr C. 2013. Ubiquitously transcribed genes use alternative polyadenylation to achieve tissue-specific expression. Genes Dev 27:2380-2396 DOI:10.1101/gad.229328.113

Love MI, Huber W, and Anders S. 2014. Moderated estimation of fold change and dispersion for RNA-seq data with DESeq2. Genome Biol 15:550 DOI:10.1186/s13059-014-0550-8

Ma L, Zhang M, Jin Y, Erdenee S, Hu L, Chen H, Cai Y, and Lan X. 2018. Comparative Transcriptome Profiling of mRNA and IncRNA Related to Tail Adipose Tissues of Sheep. Front Genet 9:365 DOI:10.3389/fgene.2018.00365

MacDonald CC. 2019. Tissue-specific mechanisms of alternative polyadenylation: Testis, brain, and beyond (2018 update). Wiley Interdiscip Rev RNA 10:e1526 DOI:10.1002/wrna.1526

Mastrangelo S, Bahbahani H, Moioli B, Ahbara A, Al Abri M, Almathen F, da Silva A, Belabdi I, Portolano B, Mwacharo JM, Hanotte O, Pilla F, and Ciani E. 2019a. Novel and known signals of selection for fat deposition in domestic sheep breeds from Africa and Eurasia. PLoS One 14:e0209632 DOI:10.1371/journal.pone.0209632

Mastrangelo S, Moioli B, Ahbara A, Latairish S, Portolano B, Pilla F, and Ciani E. 2019b. Genome-wide scan of fat-tail sheep identifies signals of selection for fat deposition and adaptation. Animal Production Science 59 DOI:10.1071/an17753

Peer) reviewing PDF | (2021:07:63246:1:1:NEW 9 Sep 2021) 
555

556

557

558

559

560

561

562

563

564

565

566

567

568

569

570

571

572

573

574

575

576

577

578

579

580

581

582

583

584

585

586

587

588

589

590

591

592

593

594

595

596

597

598

599

600

601

602

603

Miao X, Luo Q, Qin X, and Guo Y. 2015a. Genome-wide analysis of microRNAs identifies the lipid metabolism pathway to be a defining factor in adipose tissue from different sheep. Sci Rep 5:18470 DOI:10.1038/srep18470

Miao X, Luo Q, Qin X, Guo Y, and Zhao H. 2015b. Genome-wide mRNA-seq profiling reveals predominant down-regulation of lipid metabolic processes in adipose tissues of Small Tail Han than Dorset sheep. Biochem Biophys Res Commun 467:413-420 DOI:10.1016/j.bbrc.2015.09.129

Moioli B, Pilla F, and Ciani E. 2015. Signatures of selection identify loci associated with fat tail in sheep. J Anim Sci 93:4660-4669 DOI:10.2527/jas.2015-9389

Moradi MH, Nejati-Javaremi A, Moradi-Shahrbabak M, Dodds KG, and McEwan JC. 2012. Genomic scan of selective sweeps in thin and fat tail sheep breeds for identifying of candidate regions associated with fat deposition. BMC Genet 13:10 DOI:10.1186/14712156-13-10

Motter MM, Corva PM, and Soria LA. 2021. Expression of calpastatin isoforms in three skeletal muscles of Angus steers and their association with fiber type composition and proteolytic potential. Meat Sci 171:108267 DOI:10.1016/j.meatsci.2020.108267

Nattrass GS, Cafe LM, McIntyre BL, Gardner GE, McGilchrist P, Robinson DL, Wang YH, Pethick DW, and Greenwood PL. 2014. A post-transcriptional mechanism regulates calpastatin expression in bovine skeletal muscle. J Anim Sci 92:443-455 DOI:10.2527/jas.2013-6978

Pan Y, Jing J, Zhao J, Jia X, Qiao L, An L, Li B, Ma Y, Zhang Y, and Liu W. 2018. MicroRNA expression patterns in tail fat of different breeds of sheep. Livestock Science 207:7-14 DOI:10.1016/j.livsci.2017.11.007

Recazens E, Mouisel E, and Langin D. 2020. Hormone-sensitive lipase: sixty years later. Prog Lipid Res 82:101084 DOI:10.1016/j.plipres.2020.101084

Rhoads A, and Au KF. 2015. PacBio Sequencing and Its Applications. Genomics Proteomics Bioinformatics 13:278-289 DOI:10.1016/j.gpb.2015.08.002

Salmela L, and Rivals E. 2014. LoRDEC: accurate and efficient long read error correction. Bioinformatics 30:3506-3514 DOI:10.1093/bioinformatics/btu538

Shannon P, Markiel A, Ozier O, Baliga NS, Wang JT, Ramage D, Amin N, Schwikowski B, and Ideker T. 2003. Cytoscape: a software environment for integrated models of biomolecular interaction networks. Genome Res 13:2498-2504 DOI:10.1101/gr.1239303

Sun J, Yang Z, Xiao P, Liu Y, Ji H, Du Z, and Chen L. 2017. Two isoforms of hormone-sensitive lipase $b$ are generated by alternative exons usage and transcriptional regulation by insulin in grass carp (Ctenopharyngodon idella). Fish Physiol Biochem 43:539-547 DOI:10.1007/s10695-016-0308-1

Sun L, Luo H, Bu D, Zhao G, Yu K, Zhang C, Liu Y, Chen R, and Zhao Y. 2013. Utilizing sequence intrinsic composition to classify protein-coding and long non-coding transcripts. Nucleic Acids Res 41:e166 DOI:10.1093/nar/gkt646

Szklarczyk D, Morris JH, Cook H, Kuhn M, Wyder S, Simonovic M, Santos A, Doncheva NT, Roth A, Bork P, Jensen LJ, and von Mering C. 2017. The STRING database in 2017: quality-controlled protein-protein association networks, made broadly accessible. Nucleic Acids Res 45:D362-D368 DOI:10.1093/nar/gkw937

Tatusov RL, Fedorova ND, Jackson JD, Jacobs AR, Kiryutin B, Koonin EV, Krylov DM, Mazumder R, Mekhedov SL, Nikolskaya AN, Rao BS, Smirnov S, Sverdlov AV, Vasudevan S, Wolf YI, Yin JJ, and Natale DA. 2003. The COG database: an updated version includes eukaryotes. BMC Bioinformatics 4:41 DOI:10.1186/1471-2105-4-41

Tian B, and Manley JL. 2017. Alternative polyadenylation of mRNA precursors. Nat Rev Mol Cell Biol 18:18-30 DOI:10.1038/nrm.2016.116

Peer) reviewing PDF | (2021:07:63246:1:1:NEW 9 Sep 2021) 
604

605

606

607

608

609

610

611

612

613

614

615

616

617

618

619

620

621

622

623

624

625

626

627

628

629

630

631

632

633

634

635

636

637

638

639

640

641

642

643

644

645

646

647

648

649

650

651

652

653

654

Vysochan A, Sengupta A, Weljie AM, Alwine JC, and Yu Y. 2017. ACSS2-mediated acetyl-CoA synthesis from acetate is necessary for human cytomegalovirus infection. Proc Natl Acad Sci U S A 114:E1528-E1535 DOI:10.1073/pnas.1614268114

Walter W, Sanchez-Cabo F, and Ricote M. 2015. GOplot: an R package for visually combining expression data with functional analysis. Bioinformatics 31:2912-2914 DOI:10.1093/bioinformatics/btv300

Wang L, Park HJ, Dasari S, Wang S, Kocher JP, and Li W. 2013. CPAT: Coding-Potential Assessment Tool using an alignment-free logistic regression model. Nucleic Acids Res 41:e74 DOI:10.1093/nar/gkt006

Wang W, Wang L, Wang L, Tan M, Ogutu CO, Yin Z, Zhou J, Wang J, Wang L, and Yan X. 2021. Transcriptome analysis and molecular mechanism of linseed (Linum usitatissimum L.) drought tolerance under repeated drought using single-molecule long-read sequencing. BMC Genomics 22:109 DOI:10.1186/s12864-021-07416-5

Wang X, Zhou G, Xu X, Geng R, Zhou J, Yang Y, Yang Z, and Chen Y. 2014. Transcriptome profile analysis of adipose tissues from fat and short-tailed sheep. Gene 549:252-257 DOI:10.1016/j.gene.2014.07.072

Wang Y, Liu J, Huang BO, Xu YM, Li J, Huang LF, Lin J, Zhang J, Min QH, Yang WM, and Wang XZ. 2015. Mechanism of alternative splicing and its regulation. Biomed Rep 3:152-158 DOI:10.3892/br.2014.407

Wei C, Wang H, Liu G, Wu M, Cao J, Liu Z, Liu R, Zhao F, Zhang L, Lu J, Liu C, and Du L. 2015. Genome-wide analysis reveals population structure and selection in Chinese indigenous sheep breeds. BMC Genomics 16:194 DOI:10.1186/s12864-015-1384-9

Wu TD, and Watanabe CK. 2005. GMAP: a genomic mapping and alignment program for mRNA and EST sequences. Bioinformatics 21:1859-1875 DOI:10.1093/bioinformatics/bti310

Xiang R, Hayes BJ, Vander Jagt CJ, MacLeod IM, Khansefid M, Bowman PJ, Yuan Z, ProwseWilkins CP, Reich CM, Mason BA, Garner JB, Marett LC, Chen Y, Bolormaa S, Daetwyler HD, Chamberlain AJ, and Goddard ME. 2018. Genome variants associated with RNA splicing variations in bovine are extensively shared between tissues. BMC Genomics 19:521 DOI:10.1186/s12864-018-4902-8

Xie C, Mao X, Huang J, Ding Y, Wu J, Dong S, Kong L, Gao G, Li CY, and Wei L. 2011. KOBAS 2.0: a web server for annotation and identification of enriched pathways and diseases. Nucleic Acids Res 39:W316-322 DOI:10.1093/nar/gkr483

Xu SS, Ren X, Yang GL, Xie XL, Zhao YX, Zhang M, Shen ZQ, Ren YL, Gao L, Shen M, Kantanen J, and Li MH. 2017. Genome-wide association analysis identifies the genetic basis of fat deposition in the tails of sheep (Ovis aries). Anim Genet 48:560-569 DOI:10.1111/age.12572

Yin Q, Yang H, Han X, Fan B, and Liu B. 2012. Isolation, mapping, SNP detection and association with backfat traits of the porcine CTNNBL1 and DGAT2 genes. Mol Biol Rep 39:4485-4490 DOI:10.1007/s11033-011-1238-8

Yuan Z, Liu E, Liu Z, Kijas JW, Zhu C, Hu S, Ma X, Zhang L, Du L, Wang H, and Wei C. 2017. Selection signature analysis reveals genes associated with tail type in Chinese indigenous sheep. Anim Genet 48:55-66 DOI:10.1111/age.12477

Yuan Z, Sunduimijid B, Xiang R, Behrendt R, Knight MI, Mason BA, Reich CM, Prowse-Wilkins C, Vander Jagt CJ, Chamberlain AJ, MacLeod IM, Li F, Yue X, and Daetwyler HD. 2021. Expression quantitative trait loci in sheep liver and muscle contribute to variations in meat traits. Genet Sel Evol 53:8 DOI:10.1186/s12711-021-00602-9

Zhang T, Gao H, Sahana G, Zan Y, Fan H, Liu J, Shi L, Wang H, Du L, Wang L, and Zhao F. 2019. Genome-wide association studies revealed candidate genes for tail fat deposition and body size in the Hulun Buir sheep. J Anim Breed Genet 136:362-370 DOI:10.1111/jbg. 12402

Peer) reviewing PDF | (2021:07:63246:1:1:NEW 9 Sep 2021) 
655

656

657

658

659

660

661

662

663

664

665

666
Zhao F, Deng T, Shi L, Wang W, Zhang Q, Du L, and Wang L. 2020. Genomic Scan for Selection Signature Reveals Fat Deposition in Chinese Indigenous Sheep with Extreme Tail Types. Animals (Basel) 10 DOI:10.3390/ani10050773

Zhi D, Da L, Liu M, Cheng C, Zhang Y, Wang X, Li X, Tian Z, Yang Y, He T, Long X, Wei W, and Cao G. 2018. Whole Genome Sequencing of Hulunbuir Short-Tailed Sheep for Identifying Candidate Genes Related to the Short-Tail Phenotype. G3 (Bethesda) 8:377383 DOI:10.1534/g3.117.300307

Zhu C, Fan H, Yuan Z, Hu S, Ma X, Xuan J, Wang H, Zhang L, Wei C, Zhang Q, Zhao F, and Du L. 2016. Genome-wide detection of CNVs in Chinese indigenous sheep with different types of tails using ovine high-density 600K SNP arrays. Sci Rep 6:27822

DOI:10.1038/srep27822 
Figure 1

Overview of bioinformatics pipeline

DDH, Dorper $\times($ Dorper $\times$ Hu sheep); DHH, (Dorper $\times$ Hu) $\times$ Hu sheep)

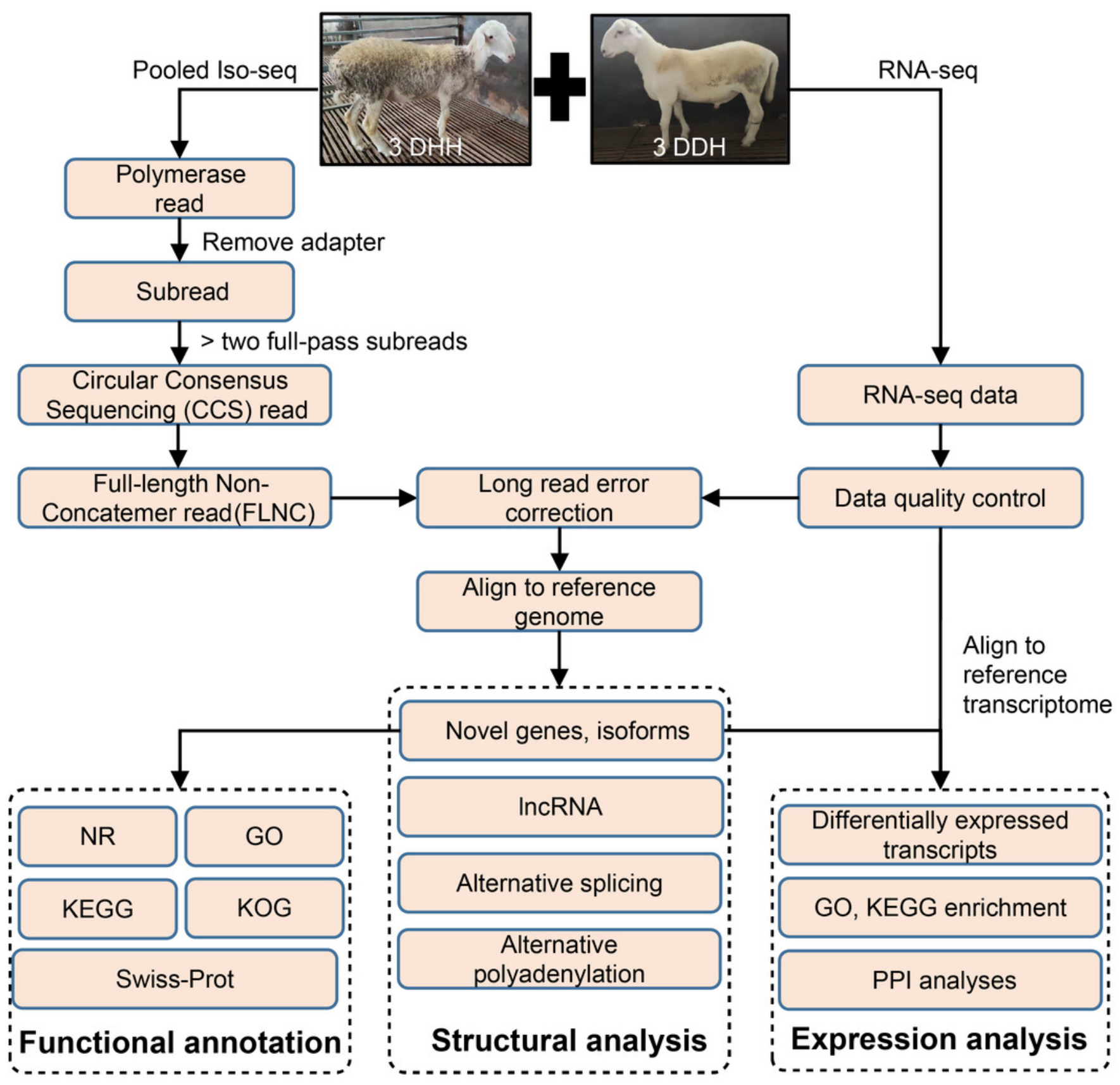


Figure 2

Genes and isoforms identified by Iso-seqencing (Iso-Seq) data

(A) Veen plot of annotated genes and identified genes by Iso-Seq data. (B) Distribution of transcribed transcripts. (C) Hist plot of three type of transcripts.

A

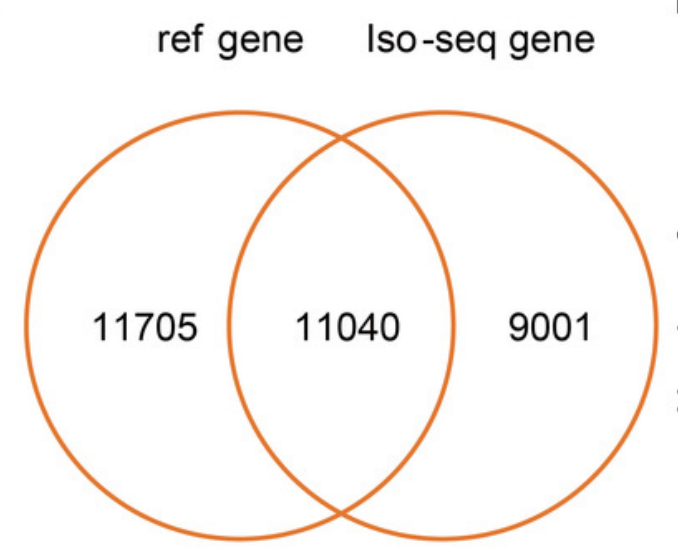

B

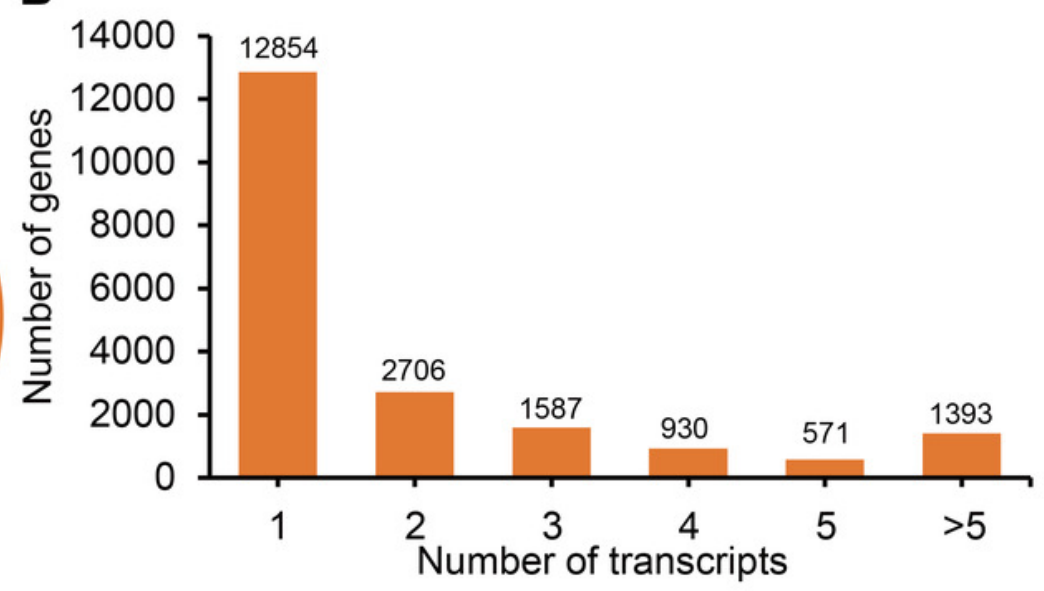

C

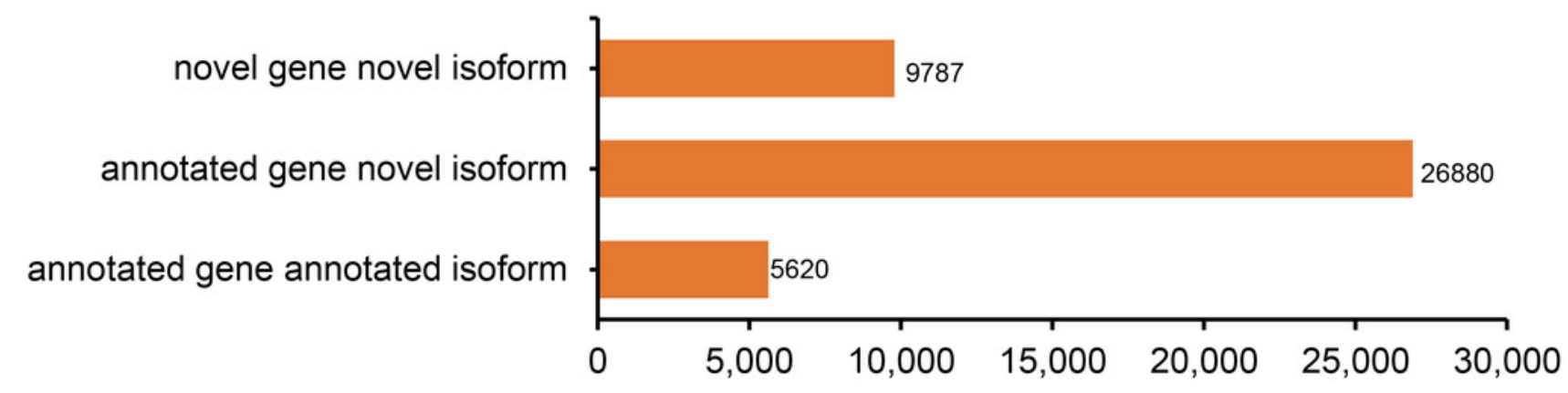


Figure 3

Veen plot of annotated transcripts

NR,NCBI non-redundant proteins; GO,Gene Ontology; KO,KEGG Orthology;KOG, Cluster of Orthologous Groups of proteins 


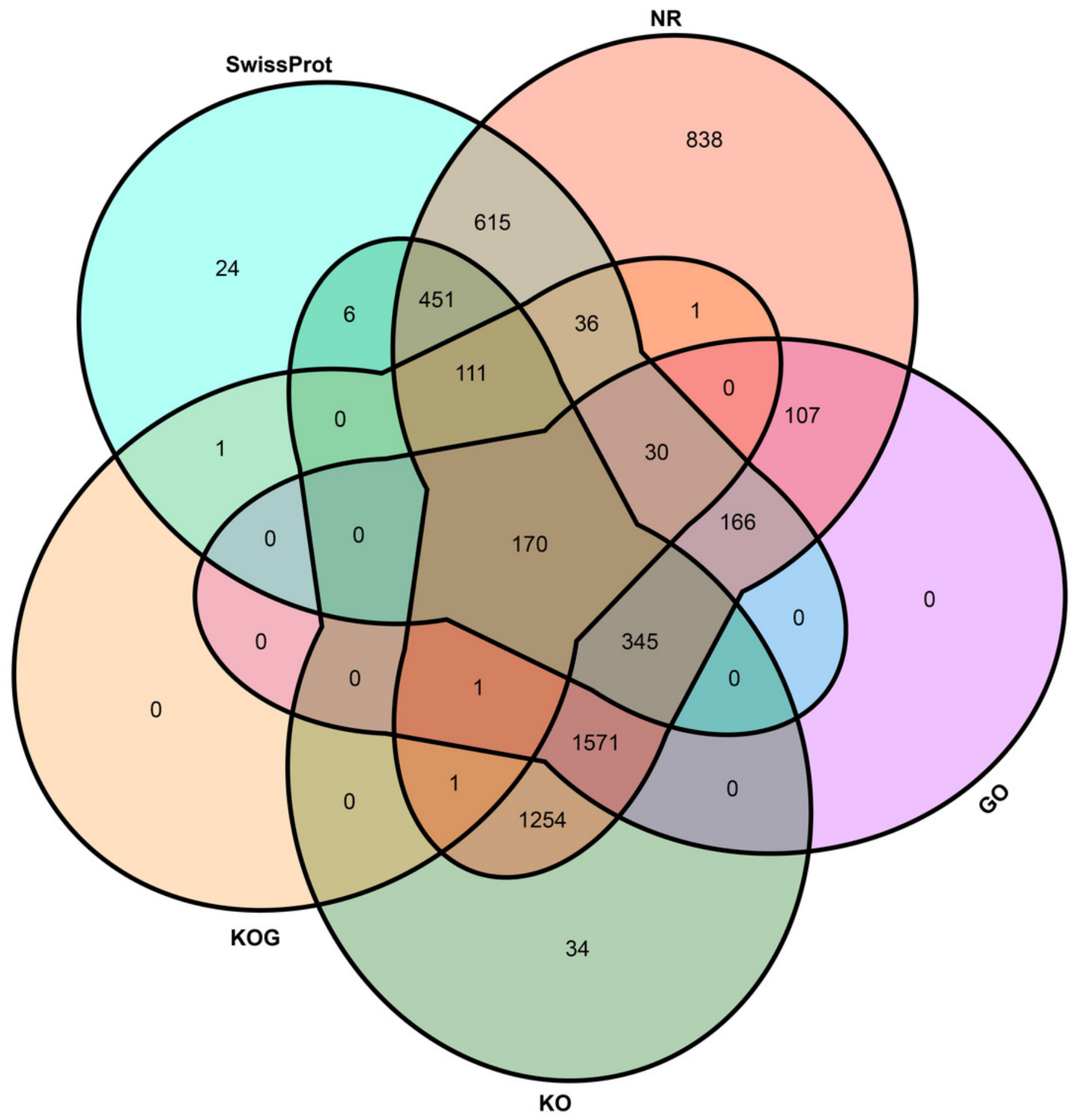


Figure 4

Identified IncRNAs

(A) Veen plot of predicted IncRNAs by four software. (B) Length distribution of predicted IncRNAs. 
A

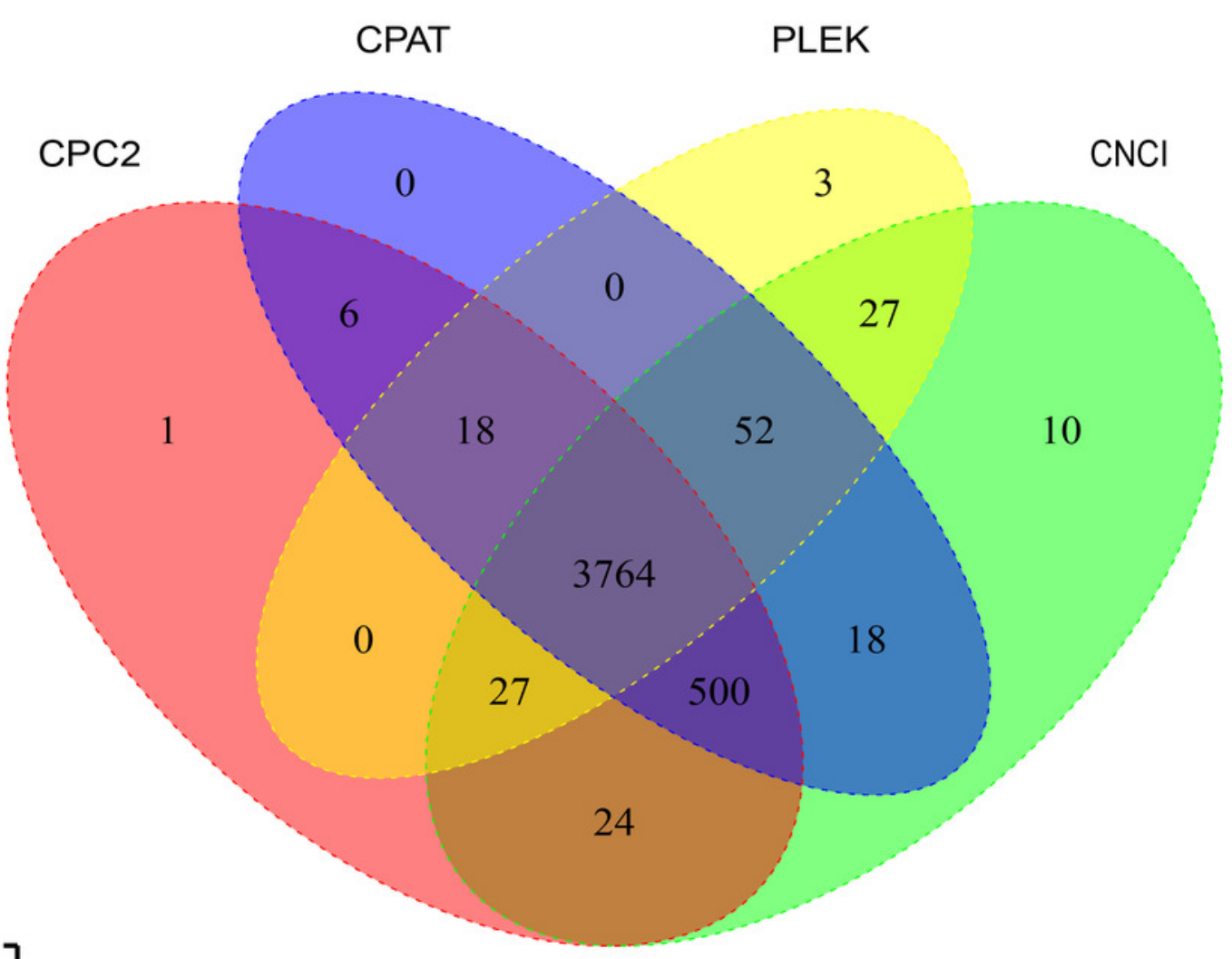

B

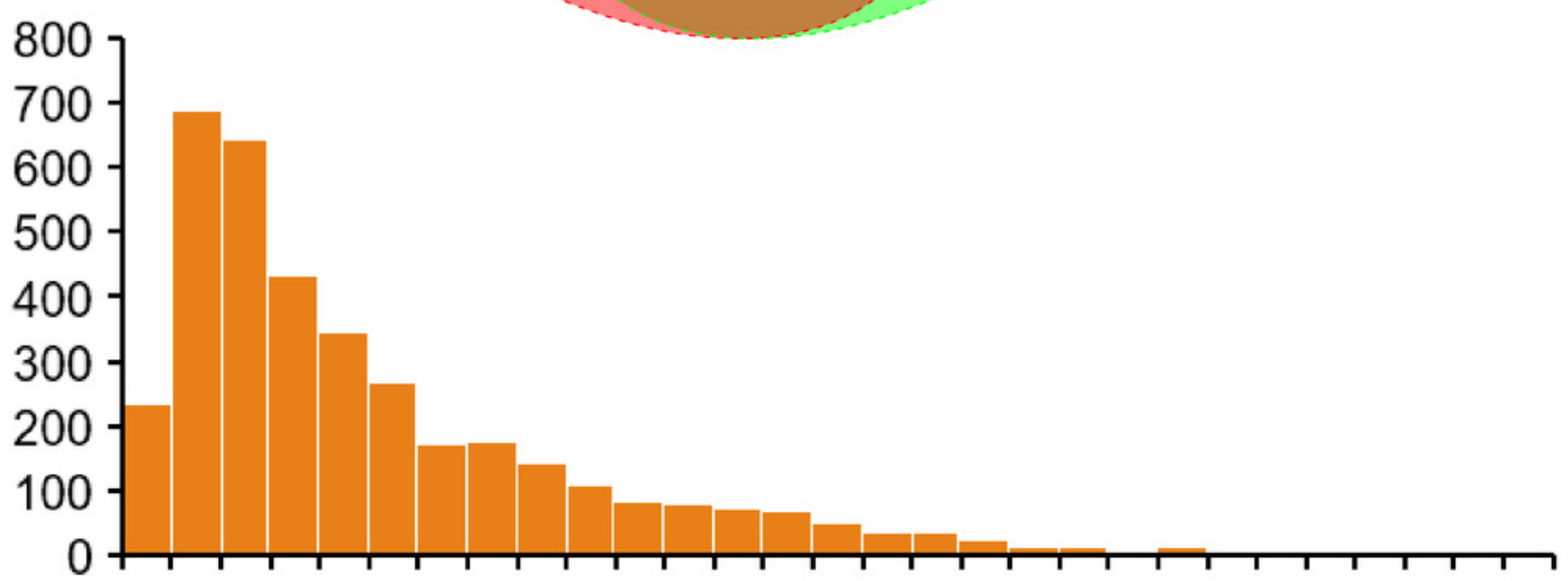

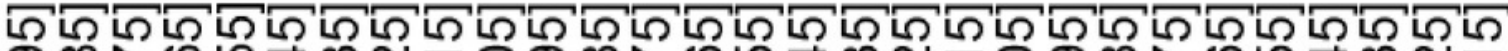
దெNG

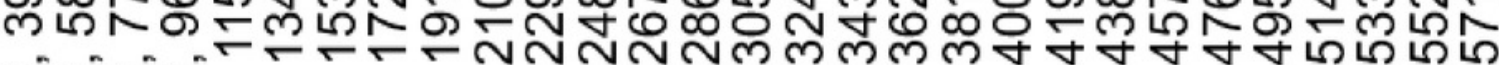
ก

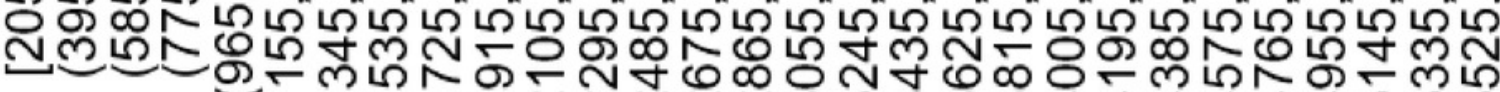

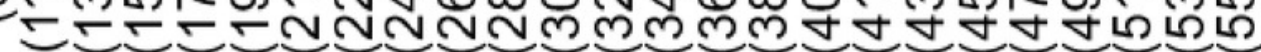


Figure 5

Identified alternative polyadenylation (APA) events.

(A) Distribution of the number of APA events. (B) APA events in ENSOARG00020025751

(ASPH). (C) APA events in ENSOARG00020017413 (unannotated) 

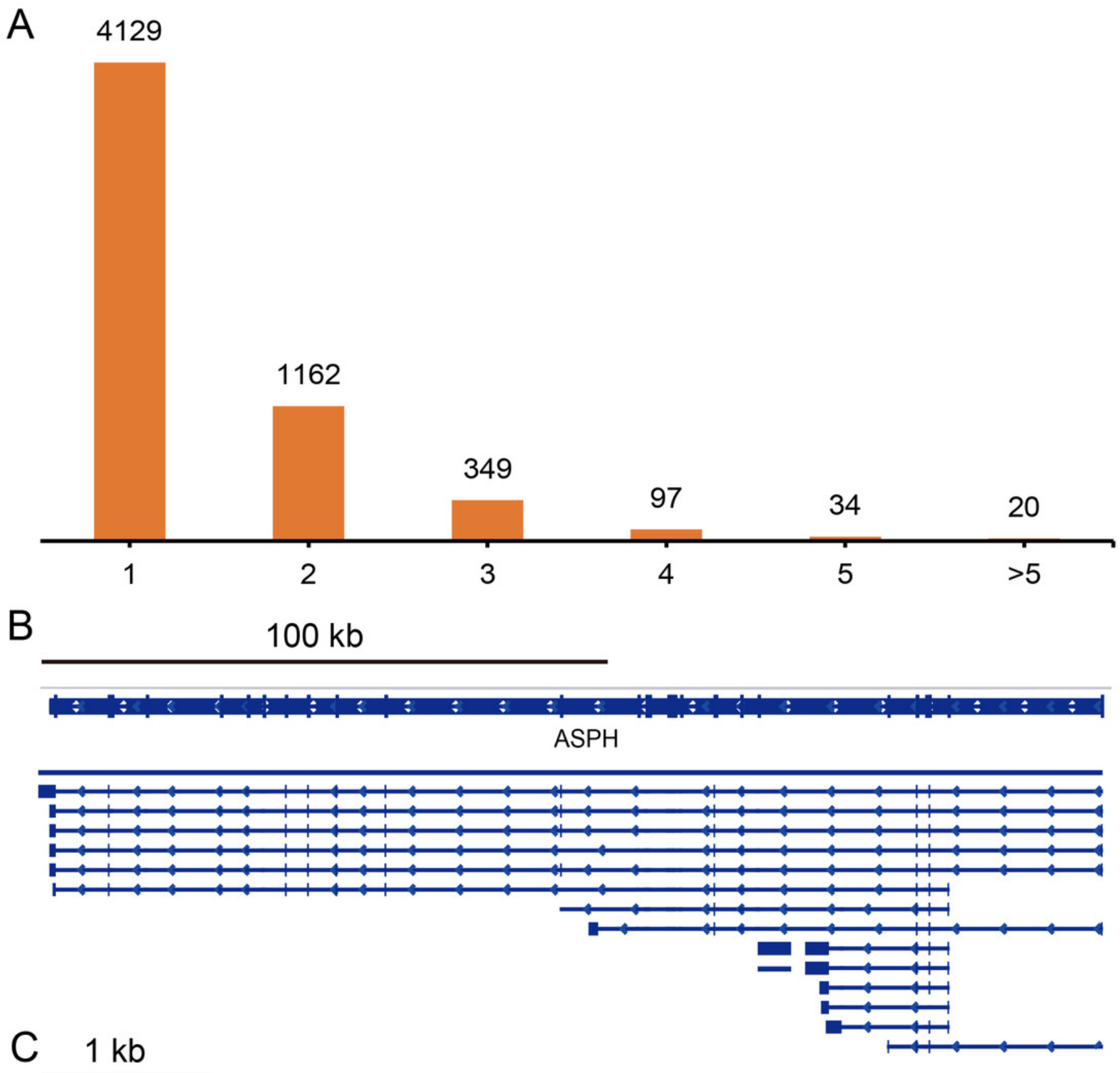

C $1 \mathrm{~kb}$
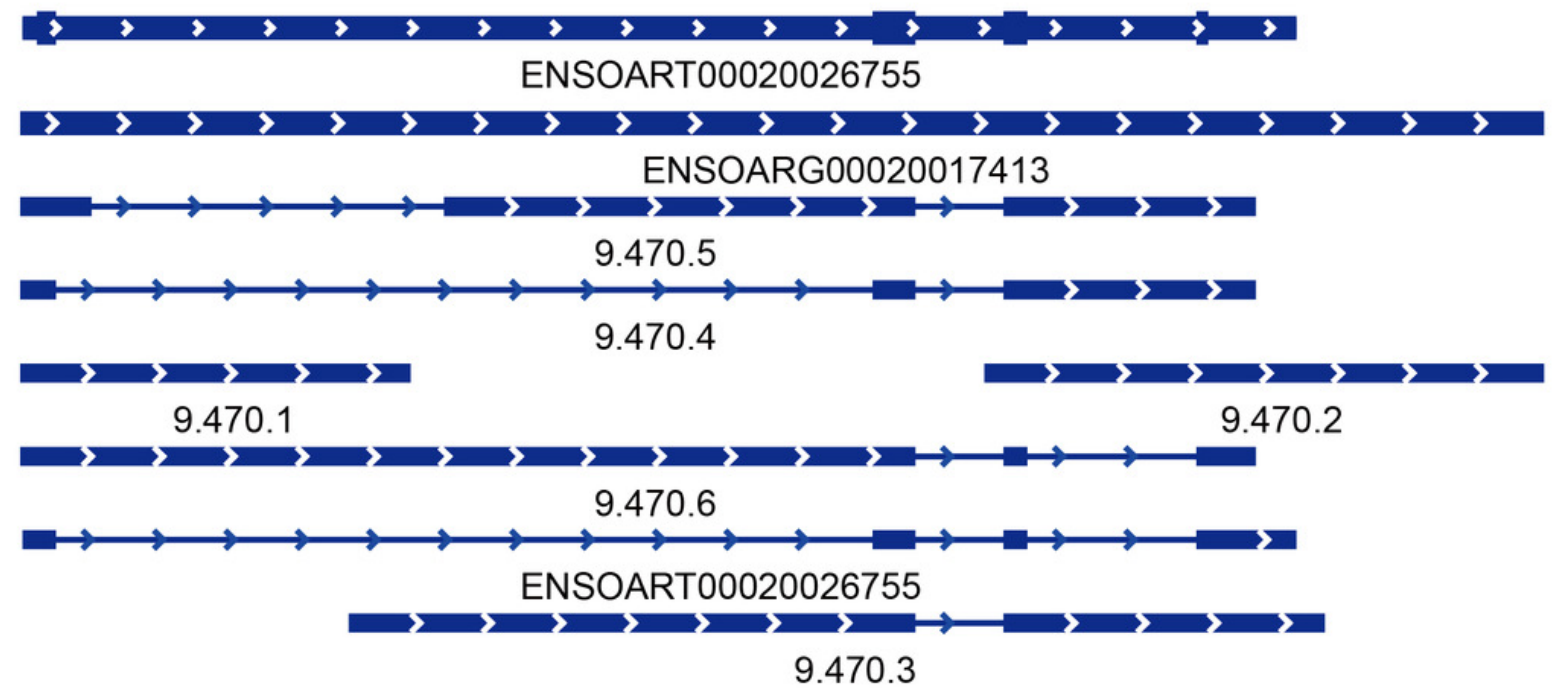

Peer) reviewing PDF | (2021:07:63246:1:1:NEW 9 Sep 2021) 
Figure 6

Classification of alternative splicing events alternative events

Exon skipping (ES)

Alternate acceptor site (AA)

3

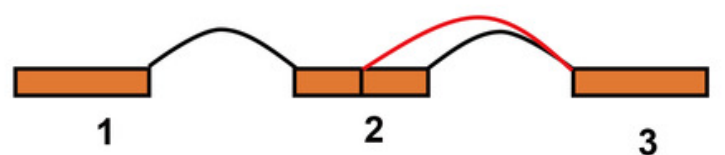

Alternate donor site (AD)

3
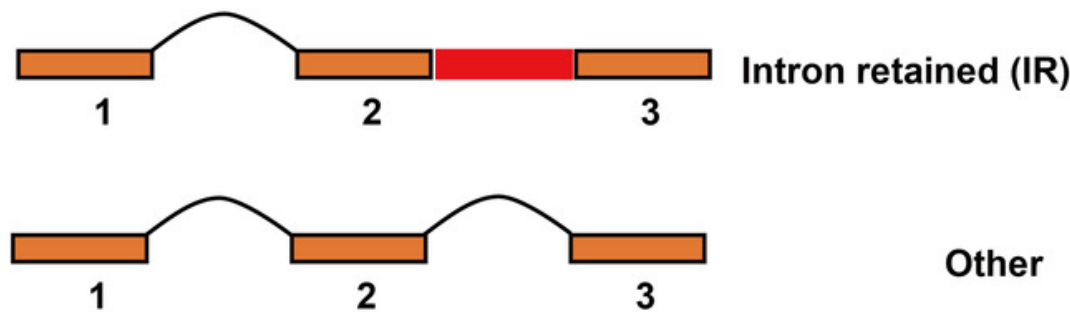

Other

6394
Number of

Ratio

4679

$26.24 \%$

$15.30 \%$

1409

$7.90 \%$

2624

$14.71 \%$

$35.85 \%$ 
Figure 7

Identified differentially expressed transcripts (DETs)

(A) Vocanno plot of DETs. (B) Correlation of differentially expressed IncRNAs (xlab) and transcripts transcribed from tail fat related candidate genes (ylab). "*" denotes $P<0.05$. "*** denotes $\mathrm{P}<0.01$. "****" denotes $\mathrm{P}<0.001$. 

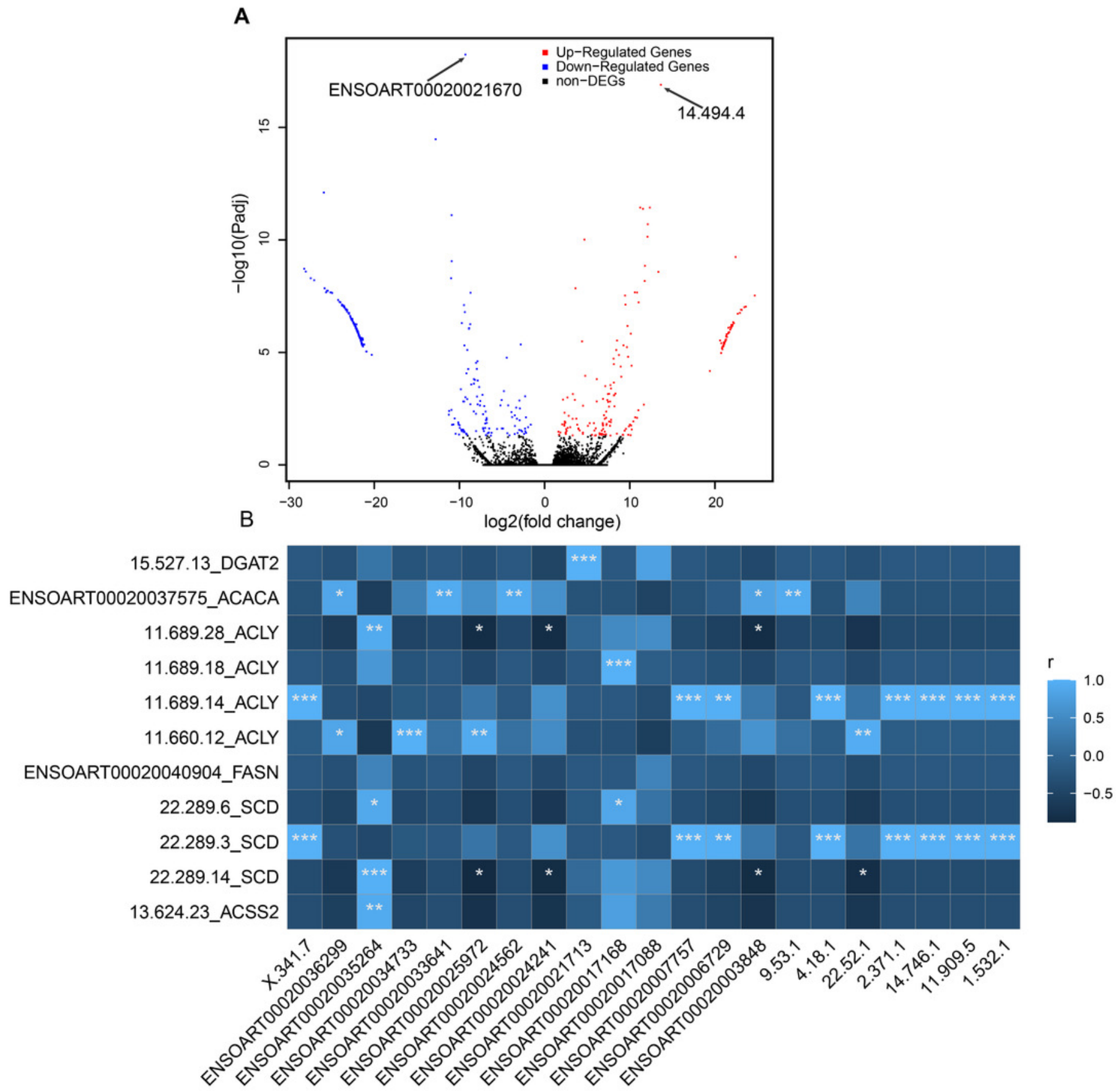
Figure 8

Functional annotation of differentially expressed transcripts (DETs).

(A) DETs interative with ENSOART00020037575 (transcribed from ACACA). (B) Gene Ontology

(GO) enrichment analysis of DETs. (C) Kyoto Encyclopedia of Genes and Genomes (KEGG) enrichment analysis of DETs.

A

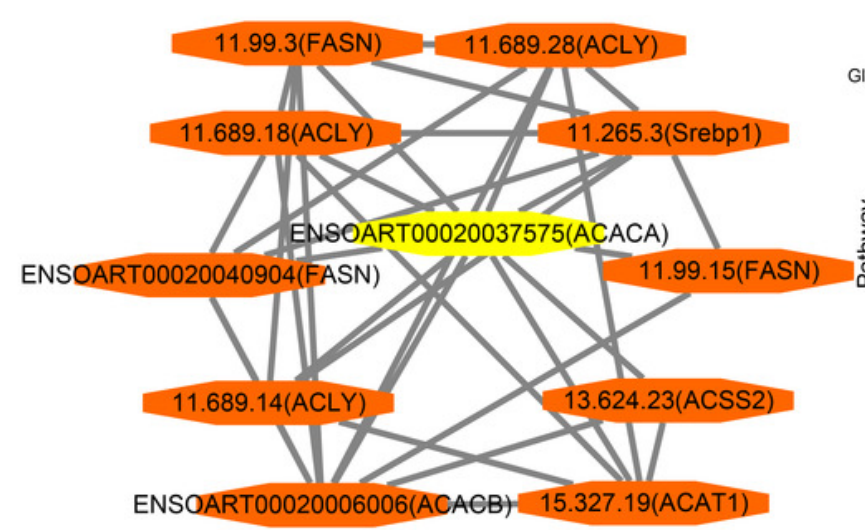

C

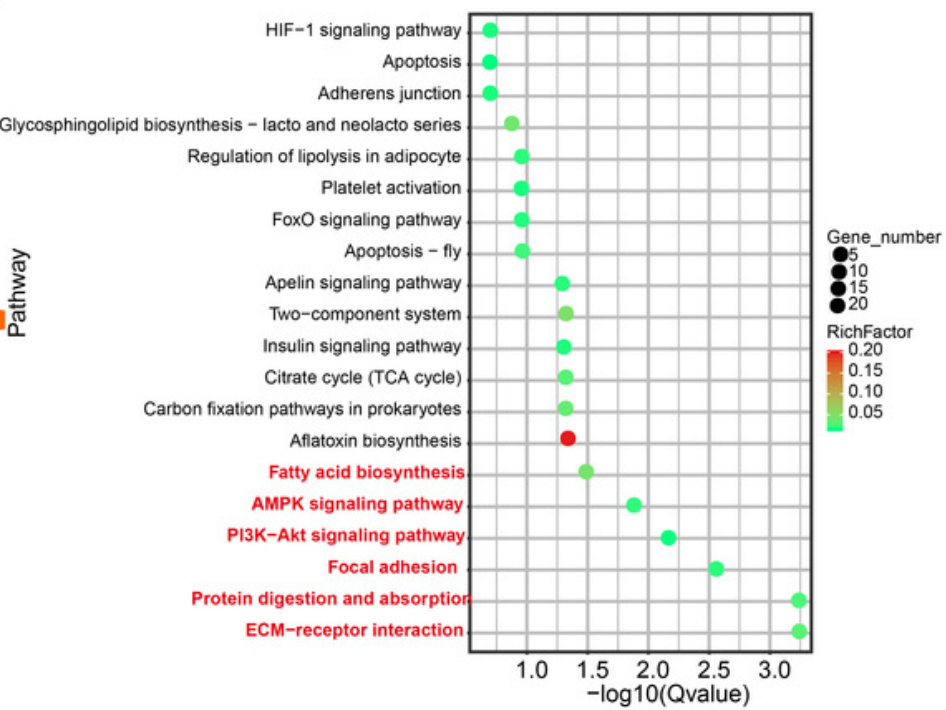

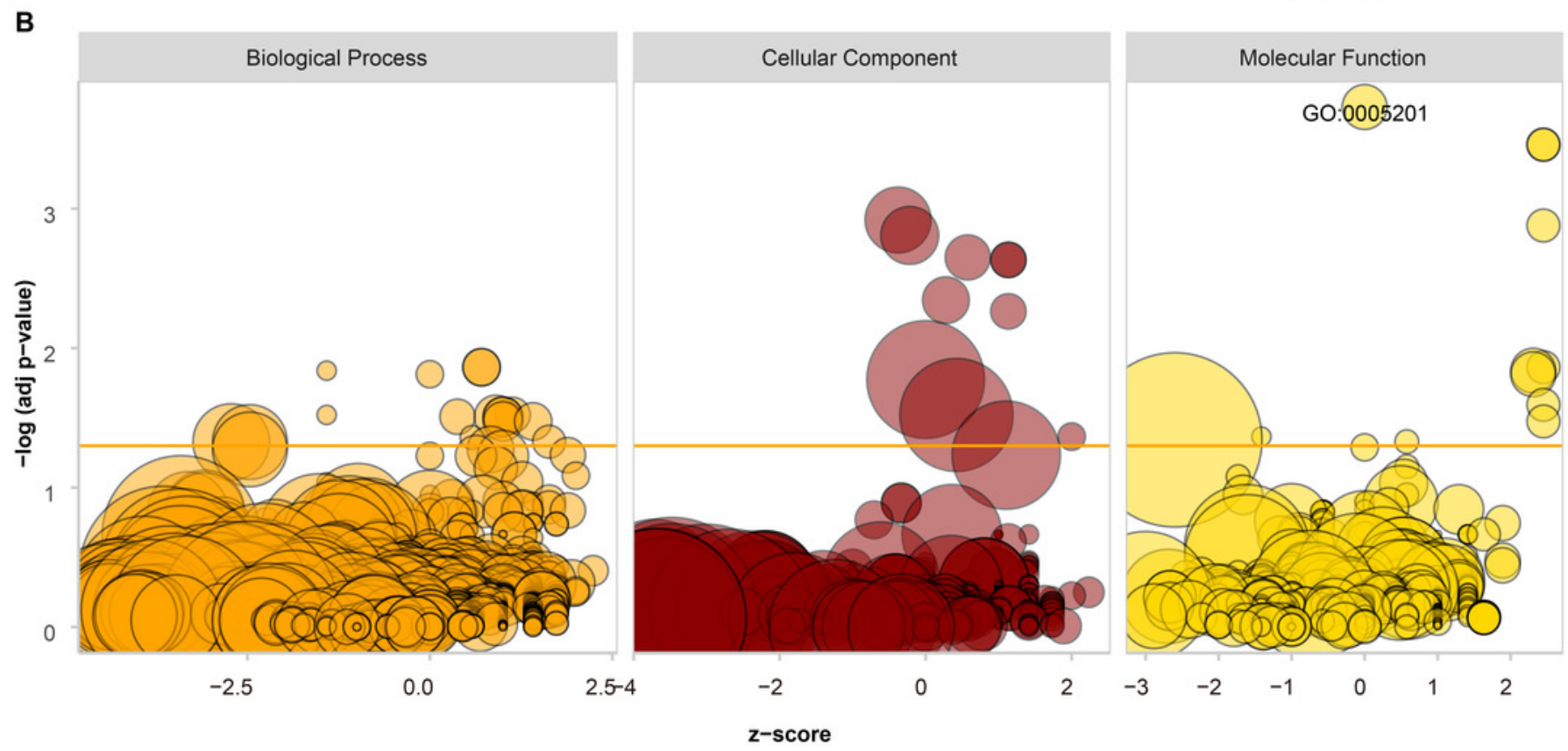


Table $\mathbf{1}$ (on next page)

Sample information 


\begin{tabular}{|c|c|c|c|c|}
\hline Cross type & $\begin{array}{l}\text { Sample } \\
\text { size }\end{array}$ & $\begin{array}{l}\text { Live weight } \\
(\mathrm{kg})\end{array}$ & $\begin{array}{l}\text { Carcass weight } \\
\qquad(\mathrm{kg})\end{array}$ & Tail fat $(\mathrm{kg})$ \\
\hline Backcross $(($ Dorper $\times \mathrm{Hu})$ & 3 & $41.53 \pm 0.95$ & $23.70 \pm 0.64$ & $0.78 \pm 0.21$ \\
\hline \multicolumn{5}{|l|}{ ×Hu sheep, DHH) } \\
\hline $\begin{array}{c}\text { Grading up }(\text { Dorper } \times \\
(\text { Dorper } \times \text { Hu sheep }), \mathrm{DDH})\end{array}$ & 3 & $40.80 \pm 4.57$ & $22.00 \pm 2.27$ & $0.11 \pm 0.03$ \\
\hline T-test $t$ value & & 0.27 & 1.27 & 5.44 \\
\hline T-test $d f$ & & 2.17 & 2.32 & 2.10 \\
\hline T-test $P$ value & & 0.8094 & 0.3161 & 0.0288 \\
\hline
\end{tabular}


Table 2 (on next page)

Summarized information of Iso sequencing (Iso-Seq) data 


\begin{tabular}{|c|c|c|c|c|}
\hline Data type & Total & Min & $\operatorname{Max}$ & Average \\
\hline & number & length & length & length \\
\hline Polymerase reads & 487,822 & 51 & 335,695 & 96,474 \\
\hline Subreads & $30,305,694$ & 51 & 250,276 & 1,474 \\
\hline Circular Consensus sequencing (CCS) reads & 360,901 & 62 & 15,123 & 2,107 \\
\hline Full-Length non-chimeric reads (FLNCs) & 271,718 & 50 & 8,211 & 1,754 \\
\hline
\end{tabular}

1 